Erschienen in: Stickel, Gerhard (Hrsg.): Sprache - Sprachwissenschaft - Öffentlichkeit. Berlin/New York: de Gruyter, 1999. S. 287-348.

(Jahrbuch des Instituts für Deutsche Sprache 1998)

DOI: https://doi.org/10.1515/9783110622645-025

\title{
Das Institut für deutsche Sprache im Jahre 1998
}

\section{Inhalt}

1. Vorbemerkungen

2. Arbeiten und Mitarbeiter der Abteilungen und Arbeitsstellen

3. Tagungen, Kolloquien und Vorträge externer Wissenschaftler am IDS

4. Lehraufträge und Vorträge von IDS-Mitarbeitern außerhalb des Instituts

5. Publikationen von IDS-Mitarbeitern

6. Kontakte des IDS zu anderen Institutionen, Studienaufenthalte und Besuche in- und ausländischer Wissenschaftler am IDS, Praktika, Besuchergruppen

7. Gremien des Instituts für deutsche Sprache

8. Besondere Nachrichten

9. Personalstärke, Anschrift, finanzielle Angaben

10. Veröffentlichungen im Jahre 1998

\section{Vorbemerkungen}

\subsection{Aufgaben und Ziele}

Das Institut für deutsche Sprache (IDS) in Mannheim besteht seit 1964. Es ist die zentrale außeruniversitäre Einrichtung zur Erforschung und Dokumentation der deutschen Sprache in ihrem gegenwärtigen Gebrauch und in ihrer neueren Geschichte. Als Mitglied der Wissenschaftsgemeinschaft Gottfried Wilhelm Leibniz (WGL) wird das IDS je zur Hälfte vom Bund und dem Land Baden-Württemberg finanziert.

In seinen Abteilungen verfolgt das Institut überwiegend längerfristige Projekte, die die Arbeit in größeren Forschungsgruppen erforderlich machen. Bei einer Reihe von Forschungsvorhaben arbeitet das IDS mit Projektgruppen und Einzelforschern aus den Hochschulen zusammen.

Bibliothek, Archive, Dokumentationen, maschinenlesbare Textsammlungen und Sprachdatenbanken stehen auch externen Wissenschaftlern zur Verfügung.

Mit seinen Vortragsveranstaltungen, Tagungen und Kolloquien ist das IDS ein Ort der wissenschaftlichen Begegnung und Kommunikation für in- und ausländische Germanisten und alle an Sprache Interessierten. 


\section{Abteilungen und Forschungsschwerpunkte}

\section{Abteilung Grammatik}

In der Abteilung werden die grammatischen Strukturen des Deutschen erfasst und beschrieben u. a. auch im Vergleich mit anderen Sprachen.

\section{Abteilung Lexik}

Bearbeitet werden lexikologische und lexikographische Aufgaben; hierzu untersucht die Abteilung definierte lexikalische Bereiche und erstellt möglichst umfassende Dokumentationen des deutschen Wortschatzes.

\section{Abteilung Pragmatik/Sprachverwendung}

Forschungsgegenstand der Abteilung sind sprachliches Handeln und sprachliche Variabilität, d.h. die Ausprägung und Entwicklung von Sprachunterschieden. Besondere Berücksichtigung findet der mündliche Sprachgebrauch.

\section{Öffentlichkeitsarbeit und Dokumentation, Bibliothek}

In der Arbeitsstelle sind die Bereiche Öffentlichkeitsarbeit und Presse, $\mathrm{Pu}$ blikationswesen, Dokumentation und Bibliothek zusammengefasst.

\section{Linguistische Datenverarbeitung (bis 30.9.1998)}

Die Arbeiten der LDV konzentrierten sich auf die Sammlung, Aufbereitung und Auswertung der für verschiedenste wissenschaftliche Aufgaben verwendbaren Textkorpora zur deutschen Sprache.

\section{Zentrale Datenverarbeitungsdienste (ZDV) (seit 1.10.1998)}

Die ZDV unterstützt die computerbasierten Arbeiten der Abteilungen durch die Bereitstellung und Pflege der erforderlichen Software.

\subsection{Allgemeines}

Die vom Wissenschaftsrat empfohlene organisatorische Umgliederung des Instituts wurde während des Berichtsjahres abgeschlossen. Auf der Aufsichtsebene wurde neben dem Stiftungsrat, dem eigentlichen 'Aufsichtsrat' des IDS, der Wissenschaftliche Beirat aus externen Wissenschaftlern eingerichtet. Die vom Minister für Wissenschaft und Kunst Baden-Württemberg berufenen neun Mitglieder kamen am 27. Juni zur konstituierenden Sitzung des Beirats zusammen. $\mathrm{Zu}$ seinem Vorsitzenden wurde Herr Prof. Dr. Heinrich Löffler von der Univ. Basel gewählt und zum stellvertretenden Vorsitzenden Herr Prof. Dr. Ludwig M. Eichinger von der Univ. Kiel (s. auch Kap. 7).

Der Wissenschaftliche Beirat befasste sich in seiner ersten und auch einer weiteren Sitzung eingehend mit dem vom Leitungskollegium erarbeiteten Plan für die Arbeiten des IDS 1998-2003. Dieser Plan wurde unter Berücksichtigung der Stellungnahme des Wissenschaftlichen Bei- 
rats vom Stiftungsrat des IDS am 5.11.1998 genehmigt. Seitdem verfuigt das Institut für seine laufenden und neuen Vorhaben nach einer evaluationsbedingten Übergangszeit wieder über eine verbindliche mittelfristige Orientierung. Teil der neuen Arbeitsplanung sind drei größere Vorhaben, die neben den anderen Projekten fortgeführt bzw. neu bearbeitet werden, und zwar eines in jeder der drei Forschungsabteilungen: in der Abteilung Grammatik ist es GRAMMIS, ein computerbasiertes Informationssystem zur deutschen Grammatik, in der Abteilung Lexik das Projekt LEKSIS, die Entwicklung eines ebenfalls computerbasierten Informationssystems zum deutschen Wortschatz, in der Abteilung Pragmatik die Erarbeitung einer „Kommunikativen sozialen Stilistik“ des heutigen Deutsch. (Näheres in den nachfolgenden Abschnitten zu den einzelnen Abteilungen.)

Teil der neuen Planung ist eine gegenüber älteren Versionen modifizierte Fassung der Richtlinien für die wissenschaftliche Arbeit des Instituts, die schon wegen der damit beabsichtigten längerfristigen Wirkungen hier wiedergegeben werden:

Richtlinien für die wissenschaftliche Arbeit des Instituts für deutsche Sprache (IDS)

(1) Das IDS hat die Aufgabe, die deutsche Sprache in ihrem gegenwärtigen Gebrauch und in ihrer neueren Geschichte wissenschaftlich zu erforschen und zu dokumentieren. Untersucht wird die deutsche Sprache in ihren verschiedenen Ausprägungen. Fragen der Sprachentwicklung, der Sprachnormung, der Sprachkritik, des Sprachkontakts und des Spracherwerbs werden berücksichtigt und zwar auch im europäischen Kontext.

(2) Das IDS verfolgt in erster Linie längerfristige Vorhaben, die umfangreiche Datensammlungen, eine größere apparative Ausstattung und eine Arbeit in Forschungsgruppen erforderlich machen. Kleinere befristete Projekte sind den größeren Forschungsvorhaben zugeordnet. Bei der Vorbereitung und Durchführung seiner Vorhaben arbeitet das IDS mit Hochschulinstituten und mit anderen Forschungseinrichtungen zusammen.

(3) Ziele der Untersuchungen sind übergreifende Darstellungen des Sprachsystems und der Sprachverwendung sowie vergleichende Beschreibungen innersprachlicher Varianten und Darstellungen des Deutschen im Vergleich mit anderen Sprachen.

(4) Das IDS nutzt die Möglichkeiten der Datenverarbeitung für seine Forschungen. Es baut maschinenlesbare Korpora zum geschriebenen und gesprochenen Deutsch auf, nutzt zu deren Auswertung Ergebnisse und Methoden der Computerlinguistik und entwickelt auch selbst Verfahren zur Korpuserschließung. Die Korpora sind Grundlage der 
linguistischen Forschungen des IDS, können aber auch von externen Partnern für wissenschaftliche $Z$ wecke verwendet werden.

(5) Die Forschungsergebnisse des IDS stehen als linguistische Grundlagen für den Unterricht in Deutsch als Muttersprache und als Fremdsprache, für die Sprachberatung, die Sprachnormung, die sprachliche Informationsverarbeitung und die Sprachtherapie zur Verfügung.

(6) Das IDS leistet wissenschaftliche Dienste für seine eigenen Forschungsvorhaben (DV-Unterstïtzung, Bibliothek, Textsammlungen, Dokumentationen, Archive). Die Dienste stehen auch Forschern und Forschergruppen außerhalb des IDS zur Verfügung.

(7) Aufgabe des IDS ist auch, Verbindungen zwischen der sprachgermanistischen Forschung im In- und Ausland herzustellen und zu erhalten. Diesem Zweck dient vor allem die Veranstaltung von Fachtagungen und Kolloquien.

1998 wurde ein weiteres Beratungsgremium des Instituts neu gebildet, der Internationale Wissenschaftliche Rat des IDS. Diesem Gremium, das vor allem dem Ausbau und der Pflege der wissenschaftlichen Kontakte des Instituts zur Hochschulforschung im In- und Ausland dienen soll, können neben den Mitgliedern des Wissenschaftlichen Beirats bis zu 50 weitere einschlägig ausgewiesene Fachleute angehören, und zwar vor allem aus dem Ausland. Aufgrund von Vorschlägen des Leitungskollegiums berief der Wissenschaftlichen Beirat die ersten 37 Mitglieder dieses neuen Gremiums (s. Kap. 7). Es wird im Anschluss an die Jahrestagung $1999 \mathrm{zu}$ seiner ersten Sitzung zusammentreten.

Unter den Personalia des Berichtsjahres ist die Berufung der neuen Leiterin der Abteilung Grammatik hervorzuheben: Frau Prof. Dr. Gisela Zifonun (bisher FU Berlin) konnte zum 1.10.1998 ihre Tätigkeit am Institut aufnehmen. Sie wurde im Wege einer gemeinsamen Berufung von IDS und Universität Mannheim gewonnen.

Als herausragendes Ereignis ist wieder die Jahrestagung des Instituts zu nennen: 1998 zum Thema „Sprache - Sprachwissenschaft - Öffentlichkeit“". Über diese und weitere wissenschaftliche Veranstaltungen des IDS wird im Anschluss an die folgenden Kurzdarstellungen der Abteilungen und Arbeitsstellen näher berichtet.

\section{Arbeiten und Mitarbeiter der Abteilungen und Arbeitsstellen \\ 2.1 Abteilung Grammatik}

Forschungsgegenstand der Abteilung sind die grammatischen Strukturen der deutschen Gegenwartssprache, auch im Vergleich mit anderen Sprachen oder in sprachtypologischer Perspektive. Nach dem Erscheinen der dreibändigen „Grammatik der deutschen Sprache“ (Dezember 1997) kon- 
zentrieren sich die Arbeiten der Abteilung zunehmend auf folgende Schwerpunktbereiche:

- Entwicklung des hypermedialen grammatischen Informationssystems GRAMMIS

- Handbuch der Konnektoren

- Grammatik des Deutschen im europäischen Vergleich

- Arbeiten der Arbeitsstelle „Graphie und Orthographie“

Daneben werden eine Reihe von Vorhaben abgeschlossen:

- Arbeiten, die unmittelbar an die "Grammatik der deutschen Sprache“ anschließen: Projekte Qualifikation und Modifikation

- Deutsche Wortbildung

- Valenzlexikon deutscher Verben VALBU

Im Rahmen des Projekts GRAMMIS (Grundlagen eines grammatischen Informationssystems) entsteht in mehreren Stufen eine MultimediaGrammatik, in der grammatisches Wissen anschaulich vermittelt wird. Es wurden die Arbeiten zu den Themenbereichen „Grammatik aus funktionaler Sicht“, ,neue Rechtschreibung“" und „Konnektoren“ fortgesetzt. Neu hinzugekommen sind konzeptionelle Arbeiten zum Themenbereich „Wortbildung“. Um aktuellen Entwicklungen im Bereich multimedialer Informationssysteme Rechnung zu tragen, wurde im Zusammenhang mit der neuen mittelfristigen Arbeitsplanung der Umstieg von Toolbook-basierten Präsentationen auf WWW-konforme Präsentationen vorbereitet. Inhaltlich wurde ein detailliertes, in Arbeitsphasen gegliedertes Konzept für das gesamte Informationsangebot von GRAMMIS entwickelt.

Im „Handbuch der deutschen Konnektoren“ sollen satzverknüpfende Elemente unterschiedlicher Wortarten (und, denn, aber, weil usw.) in ihren syntaktischen und semantischen Gebrauchsbedingungen beschrieben werden. Für das „Handbuch der Konnektoren“" wurden im Berichtsjahr die Abschnitte zu den linguistischen Grundlagen der Konnexion und den syntaktischen Konnektorenklassen vervollständigt, überarbeitet und komprimiert sowie die Verzahnung zwischen den einzelnen Abschnitten verbessert. Sukzessive erfolgte die Konversion in die elektronische Version des Handbuchs im Rahmen von GRAMMIS.

Die im Verlauf der Arbeiten am Handbuch der Konnektoren erstellte Bibliografie zu den Konnektoren wurde im Datenbank-Format weitergeführt, komplett verschlagwortet und ist nun mit ca. 900 Einträgen als abfragbare, erweiterbare Datenbank auf der Service-Seite der IDS-Homepage allgemein zugänglich.

Nach dem Arbeitsbeginn der neuen Abteilungsleiterin im Oktober 98 wurde damit begonnen, die Konzeption für ein Vorhaben ,Grammatik des Deutschen im europäischen Vergleich" zu entwickeln. Ziel des Vorhabens 
ist eine Profilierung der grammatischen Eigenschaften des Deutschen vor dem Hintergrund der entsprechenden Optionen in anderen europäischen Sprachen. Dabei ist an eine eingehendere Kontrastierung mit europäischen Sprachen wie Englisch, Niederländisch, Französisch, Spanisch, Russisch oder Polnisch, Ungarisch oder Finnisch und eine zusätzliche Einbeziehung anderer Sprachen gedacht. Das Projekt soll auch als Grundlagenwerk für den Bereich „Deutsch als Fremdsprache“ entwickelt werden. Zunächst wird mit zwei Pilotstudien zum Genus des Substantivs und zu den Pronomina begonnen.

Im Bereich „Graphie und Orthographie“" wurde die Arbeit an dem Projekt, ,Orthographiedarstellungen im 19. Jahrhundert" fortgesetzt.Zum Ende des Jahres hin wurde mit der Auswertung der durchgeführten Analysen, und zwar bezogen auf den Wörterteil der Orthographiewerke, begonnen.

Begonnen wurde auch mit der Einrichtung eines Archivs zur Geschichte der Orthographie und der Reformbemühungen.

In dem Projekt "Quantifikation", in dem Ausdrücke zur Bezeichnung für Quantität und Verfahren für deren Bestimmung beschrieben werden (z. B. es gilt für alle, für einige, für viele) und in dem Projekt „Modifikation", in dem vor allem Adjektivattribute und Adverbiale beschrieben werden (z. B. das rote Auto, Hans rennt schnell) wurde an Monographien weitergearbeitet, die Aspekte der "Grammatik der deutschen Sprache“ vertiefen und formal präzisieren.

Das Buchmanuskript „Deutsche Wortbildung in Grundzügen“ ist nach dem Abschluss redaktioneller Arbeiten in Druck gegangen und wird Anfang 1999 in der Reihe „Schriften des Instituts für deutsche Sprache“ erscheinen.

Bis zum Ausscheiden des Mitarbeiters im Frühjahr 1999 soll eine kleine Studie zur kontrastiven Wortbildung Deutsch - Ungarisch vorgelegt werden.

Die Ausarbeitung des „Valenzlexikons deutscher Verben“ (VALBU), das primär für die Anwendung im Bereich Deutsch als Fremdsprache gedacht ist und das neben Informationen zur Valenz u. a.morphosyntaktische und semantische Erläuterungen, Hinweise zu Wortbildung, Stil, Phraseologie und zahlreiche z.T. beleggestützte Verwendungsbeispiele bereitstellt, wurde fortgesetzt. Ein größerer Teil der Manuskriptfassungen der 614 bearbeiteten Verben wurde überarbeitet und endredigiert. Mit der Abfassung von Textbausteinen zur Einleitung in das Wörterbuch wurde begonnen. Im Rahmen längerer Arbeitsbesuche der ausländischen Partner wurde die Zusammenarbeit zur Entwicklung von zweisprachigen Versionen von VALBU oder verwandter kontrastiver Valenzwörterbücher fortgesetzt. Der Sammelband mit den Ergebnissen des 1994 abgeschlossenen deutsch-französischen Projekts zur Valenz abstrakter Nomina ist als Band 10 der Reihe „Studien zur deutschen Sprache“ erschienen. 
Mitarbeiter der Abteilung:

Abteilungsleiter/Abteilungsleiterin:

Prof. Dr. Bruno Strecker (kommissarisch) (bis 30.9.1998) - Prof. Dr. Gisela Zifonun (seit 1.10.1998)

Wissenschaftliche Mitarbeiter:

Prof. Dr. Joachim Ballweg - Dr. Ursula Brauße - Dr. Eva Breindl-Hiller - Dr. Elke Donalies - Helmut Frosch - Dr. Klaus Heller - Ursula Hoberg - Jacqueline Kubczak - Dr. Wolfgang Mentrup-Wenzel - Prof. Dr. Wolfgang Motsch - Dr. Renate Pasch - Vera de Ruiter - Renate Schmidt - Dr. Rosemarie Schnerrer - Helmut Schumacher - Dr. Angelika Storrer - Prof. Dr. Bruno Strecker - Klaus Vorderwülbecke

Sekretärinnen: Ruth Maurer - Karin Laton

\subsection{Abteilung Lexik}

Die Abteilung Lexik betreibt lexikologische und lexikographische Projekte mit dem doppelten Ziel (1) der Schaffung eines modernen, allgemein zugänglichen, elektronischen lexikalisch-lexikologischen Informationssystems (LEKSIS) und (2) der Erforschung ausgewählter Wortschatzbereiche, um Verwendungsbedingungen sowie historische, neue und neueste Veränderungsprozesse der deutschen Sprache linguistisch angemessen und korpusgestützt zu erfassen. Zugleich sollen in solchen Untersuchungen tragfähige linguistische Konzepte entwickelt, erprobt und vorgestellt werden. Die Ergebnisse der dafür geeigneten Arbeiten sollen grundsätzlich als Systemkomponenten auch über LEKSIS abrufbar sein. Die Korpusbasis für alle Untersuchungen wird auf der Grundlage der vorhandenen Korpora aufbereitet bzw. im Zusammenwirken der Abteilung mit den anderen Abteilungen und den zentralen DV-Diensten des IDS geschaffen.

- Lexikologische und lexikographische Projekte

\section{- LEKSIS:}

An der Konzeption und Überarbeitung des neuen, herkömmliche Projektgrenzen übergreifenden Vorhabens LEKSIS (lexikalisch-lexikologisches, korpusgestütztes Such- und Informationssystem) wurde im Berichtsjahr weitergearbeitet. Hier sollen von einer Internet-(Hypertext-)Oberfläche aus Recherchen in einer Datenbank möglich sein, in die Forschungsergebnisse möglichst vieler Projekte der Abteilung Lexik sowie ggf. weiterer Projekte des IDS eingehen. Es wurden Kooperationspartner, Soft- und Hardware-Lösungen gesucht und ein Drittmittelantrag gestellt. Seit Oktober 1998 wird an einem Datenmodell gearbeitet, das eine für Hypertext geeignete, benutzerfreundliche Strukturierung der Informationen erlaubt. Das Forschungsprojekt LEKSIS hat ein computergesteuertes umfassendes In- 
formationssystem zum deutschen Wortschatz zum Ziel. Es soll mittelfristig neben die vorhandenen großen Wörterbücher treten, diese ergänzen und teilweise auch ersetzen. D.h. es ist vorgesehen, alle Wörter der deutschen Gegenwartssprache mit Erläuterungen im Internet abrufbar zu machen. (Verantwortlich: U. Haß-Zumkehr)

- Neologieforschung:

Weitergeführt wurde 1998 die Materialsammlung und -aufbereitung für ein Vorhaben, das in einem Zeitraum von etwa 5 Jahren die deutschen Neologismen (Neulexeme und in gewissem Umfang auch Neubedeutungen) der Neunzigerjahre erfassen, beschreiben und dokumentieren soll, soweit sie sich im allgemeinsprachlichen Teil des Wortschatzes der deutschen Standardsprache in den Neunzigerjahren etabliert haben.

Die Ergebnisse der Neologieforschung werden als elektronische Datenbank im Rahmen von LEKSIS abrufbar sein. Das Informationsangebot zu den einzelnen Stichwörtern wird neben Bedeutungsangaben, Kollokationen und einem Belegteil Angaben zum jeweiligen Typ des Neologismus, zur Herkunft, zur Gebrauchsspezifik und zur Wortbildung umfassen.

(Verantwortlich: D. Herberg)

- Erklärende Synonymik kommunikativer Ausdrücke des Deutschen (ESKA):

Im Projekt „Erklärende Synonymik kommunikativer Ausdrücke des Deutschen" (ESKA) wurden die Verbgruppen der Repräsentativa, Kommissiva und Direktiva weiter bearbeitet. Die Deklarativa und Expressiva werden zur Zeit überarbeitet. Bei der Deutschen Forschungsgemeinschaft wurde zur personellen Verstärkung ein Antrag für ein Projekt „Tendenzen der Lexikalisierung kommunikativer Konzepte" gestellt. In Kooperation mit der Fakultät für Mathematik der TU Darmstadt (Prof. Dr. R. Wille) wurde ein TOSCANA-Format zur Erkundung der Argumentstrukturen der Verben erarbeitet. Das Manuskript des Forschungsberichts „Synonymie und Synonymik" wird bis zum Jahresende fertig gestellt.

(Verantwortlich: G. Harras)

- Korpusbasiertes Kollokationswörterbuch Französisch-Deutsch:

Weiterhin in der Vorbereitungsphase befindet sich das deutsch-französische Projekt zu „Kollokationen im Kontext“", das auf der Basis großer Parallelkorpora (deutsche Texte mit französischen Übersetzungen und französische Texte mit deutschen Übersetzungen) frequentielle Erhebungen durchführen und typische Verwendungswesen zentraler Kollokationen beschreiben wird. Das Kollokationswörterbuch ist der erste Schritt zur einer deutsch-französischen Übersetzungsplattform. Als Pilotprojekt zum Gesamtvorhaben ist inzwischen ein korpusbasiertes Kollokationswörterbuch Adjektiv/Substantiv für das Deutsche fertiggestellt worden.

(Verantwortlich: W. Teubert) 
- Traditionen des Formulierens

Die Ergebnisse des Teilprojekts 'Formulierungstraditionen in Zeitungsnachrichten des 17. bis 20. Jahrhunderts' (U. Haß-Zumkehr) werden Ende 1998 im Druck vorliegen. Das zweite Teilprojekt 'Formulierungsvariation' (H. Schmidt) soll 1999 abgeschlossen werden.

(Verantwortlich: H. Schmidt)

- Deutsches Fremdwörterbuch:

1997 ist der dritte Band der Neubearbeitung des historischen „Deutschen Fremdwörterbuchs" erschienen. Die Arbeiten zum vierten Band stehen kurz vor dem Abschluss, so dass dieser voraussichtlich 1999 erscheinen kann.

(Verantwortlich: G. Strauß)

- Frühneuhochdeutsches Wörterbuch:

Im Rahmen der 1993 begonnenen Beteiligung am „Frühneuhochdeutschen Wörterbuch" (Anderson, Goebel, Reichmann) liegen von Bd. 4 (b/p 2. Hälfte) die Lieferungen 1 und 2 vor, an der Lieferung 3 wird gearbeitet (J. Schildt). Für den Bd. 6 (f/v) (E. Link) wurde die Feinsortierung vorgenommen, erste Artikel sind ausgearbeitet.

(Verantwortlich: J. Schildt)

- Lehn-Wortbildung:

Die Ergebnisse des beendeten Projekts werden veröffentlicht. Dargestellt wird, in welchem Umfang deutsche Wortbildungen auf entlehnte Wortbildungsmuster und entlehnte Fremdmorpheme zurückzuführen sind, so z. B. die in der Gegenwartssprache modischen Bildungen mit $E x$ - auf ein Wortbildungsmuster, das im späten 18. Jahrhundert mit dem Exjesuiten einsetzte, oder wie die modernen Diskothek, Spielothek oder Videothek und Vinothek auf das Muster Bibliothek zurïckgehen und wie auch in den Reihen mit Neo-, Post- oder Inter- und Trans- geradezu massenhaft Neubildungen nach heute im Deutschen aktiven Wortbildungsmustern erzeugt werden. Die Arbeiten an den Bänden EX (G. Hoppe) und INTER/TRANS (I. Nortmeyer) sind weitgehend abgeschlossen. Ihr Erscheinen zu Anfang des Jahres 1999 ist gesichert. Die Druckvorbereitung der Bände NEO/POST/PRÄ (M. Kinne) und (O)THEK (G. Hoppe) ist weit fortgeschritten. Mit der Studie von G. Hoppe über das Wortbildungsmuster 'Ethnika + OPHON(E)' (frankophon $\mathrm{u}$. ä.) wurde in diesem Jahr die neue Reihe des IDS amades (Arbeitspapiere und Materialien zur deutschen Sprache) eröffnet.

- Lexikographische Erschließung des Wendekorpus:

Das Wörterbuch mit dem Titel „Wörter in Texten der Wendezeit - Ein alphabetisches Wörterbuch zum Wendekorpus des IDS 1989/90“" erschließt in ca. 1850 Stichwörtern wendetypische Themen und Diskurse der im IDS 
geschaffenen repräsentativen, elektronisch nutzbaren Textsammlung aus den Jahren der deutschen Wiedervereinigung. Das Wörterbuch macht ihren Gebrauch durch Kommentare bzw. Belege - oft nach Sprechergruppen und Phasen differenziert - nachvollziehbar. Die redaktionellen Arbeiten sind abgeschlossen (2 Bände plus Häufigkeitstabellen). Die Arbeiten am Layout beginnen im Januar 1999. Abschluss: 2. Quartal 1999.

Eine parallel zum Wörterbuch erarbeitete Bibliografie zu Sprache und Kommunikation in Deutschland seit der Wende (,Wende-Bibliografie") ist redaktionell abgeschlossen; sie erfasst die wissenschaftliche und (teilweise) populärwissenschaftliche Literatur, Dokumentationen und Nachschlagewerke sowie angrenzende Gebiete von Anfang 1990 bis Mitte 1998. Sie erscheint in der Reihe amades des IDS.

(Verantwortlich: M. W. Hellmann)

- Korpusaufbau und Korpustechnologie

- Korpustechnologie

Die Arbeitsgruppe Korpustechnologie ist der Abteilung Lexik zum 1. Oktober 1998 zugeordnet worden. Ihr obliegen wichtige Aufgaben der Weiter- und Neuentwicklung im IDS genutzter Korpustechnologien, insbesondere geschriebener Texte. Sie befasst sich mit der Erschließung, Analyse, Dokumentation, Pflege und Erweiterung der computerlesbaren IDS-Korpora und entwickelt textorientierte Erschließungsmethoden und darauf basierende Analysetechniken. Die meisten der bis jetzt vorliegenden Leistungen haben die Mitarbeiter der Arbeitsgruppe noch im Rahmen der ehemaligen Zentralen Arbeitsstelle Linguistische Datenverarbeitung erbracht.

Die IDS-Korpora mit einem Gesamtumfang von nunmehr 333 Mill. Wörtern beinhalten belletristische, populärwissenschaftliche und wissenschaftliche Texte, eine große Anzahl von Zeitungstexten sowie eine breite Palette weiterer Textarten aus Vergangenheit und Gegenwart. Sie sind mit dem IDS-Computersystem COSMAS I (Corpus Storage, Maintenance and Access System), das hauptsächlich auf linguistische Belange zugeschnitten ist, recherchierbar und stellen ein unverzichtbares Instrumentarium nicht nur für die linguistische Forschung am IDS, sondern durch die zunehmende Zahl von On-line-Nutzern für die nationale und internationale Germanistikforschung dar. Sie werden aber auch in wachsendem $\mathrm{Maße}$ für interdisziplinäre Untersuchungen genutzt, etwa in den Fachgebieten Psychologie, Neurologie, Kognitionswissenschaft, Sprachtherapie, Kommunikations- und Medienwissenschaft, Statistik.

Im Berichtszeitraum erfolgten

- COSMAS-I-online-Nutzungen von Januar bis Oktober 1998 über die WWW-Version: ca. 4500 (davon ca. 4000 durch externe Benutzer) über 
die alphanumerische Version: ca. 6500 (davon ca. 2500 durch externe Benutzer)

- Erweiterung des über COSMAS I verfügbaren Korpusangebots um

- die Berliner Tageszeitung taz, die tageszeitung (Sept. 1986 - Jan. 1998; ca. 155 Mill. Wörter),

- morphosyntaktisch annotierte Korpora (ca. 9,3 Mill. Wörter),

- das Magazin Lufthansa Bordbuch / Deutsch (ca. 0,24 Mill. Wörter);

- Fertigstellung des Parallelkorpus Magazin Lufthansa Bordbuch Deutsch/ Englisch (ca. 0,5 Mill. Wörter);

- Erarbeitung von Quellenbibliographien und Analysen für Programmierarbeiten zur Konvertierung externer Datenformate in COSMAS-angepasste für folgende kontinuierlich eingehende und vollautomatisch zu behandelnde Texte:

- Wochenzeitung Die Zeit (ab Jan. 1995; ca. 6 Mill. Wörter pro Jahrgang),

- Tageszeitung Mannheimer Morgen (ab Nov. 1997; ca. 11 Mill. Wörter pro Jahrgang),

- Tageszeitung Berliner Zeitung (ab Okt. 1997; ca. 16 Mill. Wörter pro Jahrgang);

- Betreuung des COSMAS-Betriebs;

- Weiterentwicklung der WWW-Schnittstelle zu COSMAS I;

- Weiterentwicklung der korpusanalytischen Methoden im Rahmen einer Studie zur korpusbasierten Lexikonvalidierung;

- Erweiterung des maschinenlesbaren Lexikons und Lemmatisierung umfangreicher Wortformenlisten;

- statistische Frequenzanalyse zur Gewinnung von Neulexemen im Projekt Neologismen;

- Fortsetzung der Arbeiten an den Quellenbibliographien im Hinblick auf automatische virtuelle Korpuskompositionen und benutzerorientierte Generierungen von Quellennachweisen $u$. a.;

- Überarbeitung früherer Korpora zwecks computativer Weiterverarbeitungen (z. B. Tagging, Konvertierung nach TEI-konformen SGML-Formaten durch die zentralen DV-Dienste des IDS);

- Organisation und Unterstützung der Nutzung der IDS-Korpora mittels COSMAS I durch in- und ausländische Wissenschaftler im Institut selbst und im Rahmen der On-line-Nutzung;

- Bearbeitung von Rechercheaufträgen, -anfragen und Weitergabe computerlesbarer Daten aus den IDS-Korpora.

(Verantwortlich: C. Belica)

- Historisches Textkorpus:

Die Arbeiten am Historischen Textkorpus zur deutschen Wortschatzentwicklung seit 1700 wurden fortgesetzt. Mit Stand Mai 1998 sind knapp 
340 Texte und Teiltexte mit insgesamt 2,2 Millionen laufenden Wörtern erfasst. Die Datenbank bildet die Grundlage zur Behandlung wortgeschichtlicher und anderer Themen der jüngeren Sprachgeschichte. Erste Ergebnisse gehen in Studien über Formulierungstraditionen seit dem 18. Jahrhundert ein. Seit Juni wird an ähnlich gegliederten Teilkorpora zur Weimarer Republik, zur nationalsozialistischen Zeit und zur frühen Nachkriegszeit gearbeitet.

(Verantwortlich: U. Haß-Zumkehr)

- Multilinguale Sprachressourcen und Verfahren

PAROLE/SIMPLE:

Das von der Europäischen Kommission geförderte Projekt LE-PAROLE (Beginn: April 1996) wurde Ende April abgeschlossen. Das Projekt diente dem Aufbau von standardisierten, vergleichbaren Sprachressourcen (elektronische Lexika mit 20.000 Einträgen, Korpora von 20 Millionen Wörtern Umfang). Beteiligt waren zentrale Sprachinstitute in 13 europäischen Ländern. Das IDS war Koordinator für den deutschen Sprachraum. Das deutsche PAROLE-Korpus hat einen Gesamtumfang von 50 Millionen Wörtern. Im Anschluss wurde mit dem Projekt SIMPLE (Semantic Information for Multilingual Plurifunctional Lexica) begonnen. Dabei werden Teile der PAROLE-Lexika semantisch bzw. konzeptuell miteinander vernetzt.

(Verantwortlich: W Teubert)

ELAN (European Language Activities Network):

ELAN verbindet die PAROLE- und TELRI-Gruppen in einem einjährigen Projekt, das durch das MLIS-Programm der Europäischen Kommission finanziert wird. Projektpartner sind die Universität Liège (Koordinator) und das IDS. Ziel ist es, standardisierte Textkorpora und Lexika über mehrerer Abfragesprachen auf verschiedenen Servern der Fachöffentlichkeit für Forschungszwecke verfügbar zu machen. Das Projekt ist zum 1. September 1998 angelaufen.

(Verantwortlich: W. Teubert)

TELRI II (Trans-European Language Resources Infrastructure):

Die zweite Phase dieses im Rahmen des COPERNICUS-Programms von der Europäischen Union geförderten Langzeitprojektes hat eine Dauer von drei Jahren; sie beginnt voraussichtlich am 1.12.98. Im TELRI-Netzwerk arbeiten inzwischen etwa vierzig bedeutende Sprachinstitute in den meisten Ländern Europas einschließlich der ehemaligen Sowjetunion auf den Gebieten Korpuslinguistik, Aufbau mono- und multilingualer Sprachressourcen und Entwicklung von Verfahren zur Extraktion von Sprachdaten aus Korpora. Im Mittelpunkt von TELRI II steht das Thema multilinguale lexikalische Semantik und der Ausbau von TRACTOR (TELRI Re- 
search Archive of Computational Tools and Resources). Daneben stehen Maßnahmen zur Förderung einer europäischen Infrastruktur für multilinguale Sprachtechnologie.

(Verantwortlich: W. Teubert)

Mitarbeiter der Abteilung:

Abteilungsleiter:

Prof. Dr. Hartmut Schmidt

Wissenschaftliche Mitarbeiter:

Dipl. Ing. Cyril Belica - PD Dr. Claudia Fraas - Prof. Dr. Gisela Harras

- PD Dr. Ulrike Haß-Zumkehr - Dr. Manfred W. Hellmann - Prof. Dr.

Dieter Herberg - Gabriele Hoppe (bis 31.1.1998) - Dr. Irmtraud Jüttner

- Dr. Heidrun Kämper - Dr. Michael Kinne - Dr. Ann Lawson - Susanne Lenz - Dr. Elisabeth Link - Dipl. rer. pol. Pantelis Nikitopoulos - Isolde Nortmeyer - Prof. Dr. Joachim Schildt - Dr. Doris Steffens - Dr. Kathrin Steyer - Dr. Gerhard Strauß - Dr. Elke Tellenbach - Dr. Wolfgang Teubert - Oda Vietze - Doris al-Wadi - Dr. Edeltraud Winkler.

Sekretärinnen:

Susanne Bergmann - Petra Brecht - Joyce-Ann Thompson - Sigrid Ziehr

\subsection{Abteilung Pragmatik}

Forschungsgegenstand der Abteilung sind sprachliches Handeln und sprachliche Variabilität, d.h. die Ausprägung und Entwicklung von Sprachunterschieden vor allem innerhalb des Deutschen. Das Aufgabenfeld umfasst im einzelnen:

- die Formen des sprachlichen Handelns und ihre Einbettung in die gesellschaftliche Kommunikationsstruktur;

- die Beziehung zwischen der Sprachverwendung und ihren medialen Bedingungen;

- die Sprachvariation im Deutschen und Sprachkontakterscheinungen in Mehrsprachigkeitssituationen unter Beteiligung des Deutschen.

Fortgesetzt wurde die Arbeit an einer umfangreichen systematischen Darstellung zur „Gesprächsrhetorik“. Gegenstand der Untersuchung sind verbale Muster und Verfahren in Problem- und Konfliktgesprächen. Das Projekt schließt an die in den vergangenen Jahren durchgeführten Projekte zu Beratungs- und Schlichtungsgesprächen sowie zur Kommunikation in der Stadt an und soll die vorhandenen gesprächsanalytischen Ergebnisse unter rhetorischer Perspektive systematisieren und vertiefen. Das Projekt wird 1999 abgeschlossen.

Abgeschlossen wird zum Jahresende das Projekt „Sprachliche Integration von Aussiedlern", das sich mit der Frage beschäftigt, wie sich Aus- 
siedler aus der GUS und Polen in die Sprach- und Kommunikationsgemeinschaft integrieren, die sie in Deutschland vorfinden. Die Forschungsschwerpunkte waren im einzelnen:

(a) Der Anpassungsprozess der Sprache der Russlanddeutschen in der Integrationsphase in Deutschland: Die Ergebnispublikation liegt bereits vor (Nina Berend: Sprachliche Anpassung. Eine soziolinguistisch-dialektologische Untersuchung zum Rußlanddeutschen).

(b) Die Zweisprachigkeit in Aussiedlerfamilien und ihre Auswirkungen auf den Spracherwerb der Kinder

(c) Die Kommunikationsbeziehungen zwischen Aussiedlern und Einheimischen und die Identitätsarbeit von Aussiedlern im Kontakt mit Einheimischen.

In der Abschlussphase befindet sich ebenfalls das Projekt „Eigenschaften gesprochener Sprache". Die theoretische Arbeit zielte darauf ab, die Variabilität gesprochener Sprache herauszuarbeiten und mündliche Kommunikation - im Vergleich mit Schriftlichkeit und technisierter Kommunikation - durch die Diskussion ihrer verschiedenartigen Grundbedingungen als eigenständiges Verständigungssystem zu verdeutlichen. Die empirischen Analysen zur Operator-Skopus-Struktur (z. B.: blo $\beta$ * ich habe keinen Schlüssel für diese Tür) haben folgende Untersuchungsschwerpunkte: Satzadverbiale in Operatorfunktion, prosodische Eigenschaften der Operator-Skopus-Struktur, Klassifizierung der Qualifizierungsleistungen von Operatoren und Stellungseigenschaften von Operatoren.

Das Pilotprojekt „Wandel im gesprochenen Deutsch" ist abgeschlossen; die Ergebnisse liegen in Aufsatzform vor. Gegenwärtig laufen die Planungen für eine breit angelegte Erhebung und Auswertung als Teil eines langfristigen Untersuchungsprogramms zum Wandel des gesprochenen Deutsch und der mündlichen Kommunikationsformen.

In Vorbereitung befindet sich ein großes Vorhaben zur kommunikativen sozialen Stilistik, das im Laufe des Jahres 1999 beginnen soll. Ziel ist die Erarbeitung einer Soziostilistik der Kommunikation in Deutschland. Im Zentrum steht der Zusammenhang zwischen der Ausprägung von kommunikativen Stilen, die als Ausdruck der sozialen Identität von gesellschaftlichen Gruppen fungieren, und sozialen Prozessen der Integration, Differenzierung, Distanzierung und Ausgrenzung. Angestrebtes Ergebnis ist eine grundlegende Darstellung von allgemeinen Stilbildungsprinzipien und Prozessmustern der sprachlich-sozialen Entwicklung, die an einer aspektreichen Auswahl von Kommunikationsvorgängen in unterschiedlichen Domänen der Gesellschaft demonstriert werden. Den Orientierungsrahmen für die einzelnen empirischen Untersuchungen bilden Dimensionen der gesellschaftlichen Gliederung, die in soziologischen und 
kulturwissenschaftlichen Arbeiten als Grundeigenschaften gesellschaftlicher Realität angesehen werden und deren Ausprägungen für die Bestimmung moderner Gesellschaften herangezogen werden:

- Vertikale Gliederung („Oben und Unten“)

- Arbeit und Freizeit

- Migration (,Ansässige und Zuwanderer")

- Lebensalter und Generationswechsel (,Jung und Alt")

- Öffentlichkeit und Privatheit

Für einzelne Teilvorhaben der Soziostilistik laufen Vorarbeiten mit einer Anschubfinanzierung aus Drittmitteln, so für die Arbeitswelt, die migrationsbedingte Mehrsprachigkeit und die Alterskommunikation.

Der Bereich Korpusausbau und Entwicklung von Korpustechnologie für Daten gesprochener Sprache ist gewachsen. Das vordringliche korpustechnologische Ziel ist die Einrichtung eines vernetzten Gesamtsystems (Eingabe, Speicherung und Zugriff) für Korpora gesprochener Sprache. Die wichtigsten Komponenten eines solchen Systems sind ein Transkriptionseditor, ein System für Text-Ton-Alignment, eine Datenbank für Transkripte und Zeitmarken, eine Verwaltungsdatenbank, ein Recherchesystem und ein Ausgabesystem für Transkripte mit Zugriff auf die Tondaten. Gegenwärtig laufen im Bereich Korpusaufbau und Entwicklung von Korpustechnologie folgende Teilprojekte, die jeweils Komponenten des Gesamtsystems der Diskursdatenverarbeitung verwirklichen:

- Die Weiterentwicklung des Transkriptionseditors DIDA für Eingabe und Ausgabe von Gesprächstranskriptionen im Zeilenblock-Format,

- die Fertigstellung von COSMAS II, das eine Weiterentwicklung von COSMAS I ist und das im Drittmittelprojekt SERGES (schriftliche Erfassung gesprochener Sprache) für die Aufnahme von Transkripten erweitert wurde,

- die Weiterentwicklung des Text-Ton-Alignments (hierzu wird ein drittmittelfinanziertes Kooperationsprojekt mit dem Institut für maschinelle Sprachverarbeitung (IMS) der Universität Stuttgart durchgeführt)

- und der Aufbau der Datenbank Gesprochenes Deutsch für die computerunterstützte Erfassung und Erschließung der Tonaufnahmen des Deutschen Spracharchivs, der Verwaltungsinformationen und der Transkripte; das Projekt ist ebenfalls drittmittelfinanziert.

Zentrale Stelle für die Archivierung und Dokumentation gesprochener Sprache ist das Deutsche Spracharchiv (DSAv). Hier sind auch die Aufgaben für den internen und externen Service angesiedelt. Die zentrale Dokumentation aller in der Abteilung verwalteten Korpora gesprochener 
Sprache wurde fortgesetzt, ebenso die Inventur der vorhandenen Begleitmaterialien der vor über 60 Jahren begonnenen Sammlung von Eberhard Zwirner. Gegenwärtig wird im Zusammenhang mit dem Projekt „Datenbank Gesprochenes Deutsch" ein umfangreiches Serviceangebot aufgebaut (Transkripte, Tonaufnahmen).

Mitarbeiter der Abteilung:

Abteilungsleiter:

Prof. Dr. Werner Kallmeyer

Wissenschaftliche Mitarbeiter:

Dr. Birgit Barden - Dr. Karl-Heinz Bausch - Frank Bodmer (ab 1.10.98) - Sylvia Dickgießer, M. A. - Dr. Mechthild Elstermann - Prof. Dr. Reinhard Fiehler - PD Dr. Inken Keim-Zingelmann - Dr. Wolfgang Klein ( $\dagger)$ - Dr. Barbara Kraft - PD Dr. Katharina Meng - Robert Neumann (ab 1.10.98) - Dipl.-Soz. Ulrich Reitemeier - Marcel Schilling, M. A. (ab 1.7.98) - Dr. Rudolf Schmidt (ab 1.10.98) - Dr. Reinhold Schmitt, M.A. - Dr. Wilfried Schütte - PD Dr. Thomas Spranz-Fogasy - Dr. Peter Wagener - Dr. Ricarda Wolf (bis 30.6.98)

Toningenieur: Wolfgang Rathke

Sekretärinnen: Doris Richter - Renate Wegener - Ulrike Willem

2.4 Zentrale Arbeitsstelle Linguistische Datenverarbeitung (LDV) (bis zum 30.9.1998)

Die Arbeitsstelle hatte drei Aufgabenbereiche:

(1) Grunddienste der Datenverarbeitung im IDS,

(2) Neu- und Weiterentwicklung von Datenverarbeitungssystemen,

(3) Erfassung und Aufbereitung der Textkorpora des IDS und korpusbezogener Service.

Die Arbeitsstelle war in eine wissenschaftliche Linguistische Datenverarbeitung und in die Grunddienste gegliedert.

\section{Die Grunddienste:}

$\mathrm{Zu}$ ihnen gehörte das Betreiben der Computer, die Pflege der Betriebssysteme, der Benutzersoftware und die Sicherung der Daten. Betrieben wurden fünf miteinander vernetzte Mehrplatz-UNIX-Computer vom Typ MX 300 (Siemens), ein UNIX-Computer vom Typ AViiON 6225 (Data General), fünf UNIX-Workstations (Sun- und Silicon Grafics-Computer) und eine Reihe von PCs. Mit dieser Ausstattung stehen im IDS rund 130 Bildschirm-Arbeitsplätze zur Verfügung. Die Grunddienste gewährleisteten auf den UNIX-Computern die Datensicherung, versorgen die Systeme mit Basis-Software und unterstützen die Benutzer in den grundlegenden Fragen der Computernutzung. 


\section{Die Linguistische Datenverarbeitung:}

Die Arbeiten waren auf die Sammlung, Aufbereitung und computative Analyse von Korpora zur deutschen Sprache zentriert. Folgende Datenverarbeitungssysteme wurden entwickelt bzw. weiterentwickelt: Auch die Abteilung „Pragmatik“ transkribiert und bearbeitet jetzt ihre Gesprächsdaten mit dem System 'Diskursdatenbank' (DIDA). Die Leistungen dieses Systems wurden so erweitert, daß auch kyrillische Zeichen und ein phonetischer Zeichensatz (IPA) zur Transkription eingesetzt werden können. Das System erlaubt die Recherche in den transkribierten Daten.

In das Korpus-Recherchensystem COSMAS wurden statistische Funktionen integriert; diese Funktionen können jetzt auch über den lemmatisierten Wörtern benutzt werden. Das COSMAS-System wurde weiter in das Internet integriert und kann jetzt auch als Web-Dienst in Anspruch genommen werden. Mit dem COSMAS-System wurden bisher über 200.000 Recherchen durchgeführt, davon über 30.000 durch externe Nutzer. Die Nutzer sind über die gesamte Welt verteilt.

Die Ergebnisse des durch die Europäische Union finanzierten Projekts 'Multilingual Enviroment for Corpusbased Lexiconbuilding' (MECOLB) wurden in dem vom Bundesministerium für Verteidigung finanzierten Projekt 'Schriftliche Erfassung gesprochener Sprache' (SERGES) weitergeführt. An diesem Projekt ist auch die Abteilung „Pragmatik“ beteiligt. Als externe Projektpartner waren der Lehrstuhl für Mustererkennung der Universität Erlangen, Prof. Niemann, der Lehrstuhl für Informatik VI der RWTH Aachen, Prof. Ney, und das Institut für Wirtschaftsinformatik in Kolding (Dänemark), Prof. Engel, in den SERGES-Projektrahmen integriert. Diese Partner bringen ihre Kompetenz im Bereich der Spracherkennung, der statistisch basierten Sprachanalyse und in der Terminologiearbeit in das Projekt ein. SERGES wiederum ist in eine wissenschaftliche Arbeitsgemeinschaft „Sprachstrukturen“ eingebettet, die aus weiteren Forschungsstellen der Industrie und von Universitäten besteht und die ihre Ergebnisse auf in regelmäßigen Abständen stattfindenden Kolloquien präsentiert. Die SERGES-Arbeiten waren in die Gesamtvorhaben der LDV des IDS eingebunden. Ziel dieser Weiterentwicklung ist ein Arbeitsplatz für die computerunterstützte Beschreibung und Analyse von Gesprächsdaten. Der Arbeitsplatz baut auf COSMAS-II (Ergebnis des EU-Projektes MECOLB) auf. COSMAS-II wird um die Integration von gesprochenem Material in digitalisierter Form weiterentwickelt; es wird die Zuordnung von digitalisierten, gesprochenen Äußerungen zu deren Transkriptionen ermöglichen. Die Recherche über den Transkripten liefert gleichzeitig die originalen Gesprächsabschnitte mit. Des Weiteren wird im SERGES-Rahmen COSMAS-II mit einer Schnittstelle versehen, die ein Spracherkennungsmodul aufnehmen kann. Zielsetzung der Integration von Spracher- 
kennung und Korpustechnologie ist die optimale Computerunterstützung der zeitaufwendigen Transkription von Gesprächen.

Im Auftrag eines namhaften deutschen Wörterbuchverlages wurde ein strikt korpusbasiertes Design für ein zweisprachiges Wörterbuch anhand der Einträge zum Buchstaben ,s" ausgearbeitet.

Mitarbeiter der Arbeitsstelle:

Leitung:

Robert Neumann

Wissenschaftliche Mitarbeiter:

Dipl.-Ing. Cyril Belica (stellvertretender Leiter) - Dr. Irmtraud Jüttner Dr. Rudolf Schmidt - Dipl.-Inf. Eric Seubert - Doris al-Wadi

Leitung der Grunddienste:

Peter Mückenmüller

Technische Mitarbeiter:

Siegmund Gruschka - Matthias Hördt - Rainer Krauß - Ingrid Schellhammer

Sekretariat:

Irmgard Schlösser

Die Arbeitsstelle wurde zum 1.10.1998 umstrukturiert. Die Grunddienste werden seitdem in der Arbeitsstelle Zentrale DV-Dienste weitergeführt. Die Aufgaben der Linguistischen Datenverarbeitung (im engeren Sinn) wurden dezentralisiert, d. h. in die Forschungsabteilungen verlegt.

\subsection{Zentrale Arbeitsstelle Öffentlichkeitsarbeit und Dokumentation}

In der Arbeitsstelle sind die Bereiche

- Öffentlichkeitsarbeit und Presse

- Publikationswesen

- Dokumentation

- Bibliothek

organisatorisch zusammengefasst.

$\mathrm{Zu}$ den laufenden Aufgaben der Arbeitsstelle zählen: Pflege von Pressekontakten, Redaktion der Zeitschrift „Sprachreport“, Redaktion der Zeitschrift „Deutsche Sprache“, Gästebetreuung, Zusammenstellen von Besucherprogrammen, Betreuung des Vereins der Freunde des IDS, Bearbeitung von Praktikumsanfragen, Tagungsorganisation, Druckvorlagenerstellung für die IDS-Publikationen, Zentrale Adressverwaltung, Vertrieb der Eigenverlagspublikationen und die Bearbeitung von Anfragen. Die Anfragen per E-Mail haben besonders zugenommen. Hinzu kommen Aktivitäten zur Verbesserung der internen Information und Kommunikation. 
Neben diesen laufenden Aufgaben war 1998 der Schwerpunkt der Öffentlichkeitsarbeit die Organisation und Durchführung der Jahrestagung zum Thema „Sprache - Sprachwissenschaft - Öffentlichkeit.“ Das Thema legte nahe, dass die Öffentlichkeitsarbeit auch an der inhaltlichen Gestaltung der Tagung beteiligt war. Auch im Jahr 1998 war das öffentliche Interesse an der Rechtschreibreform groß. Durch das Urteil des Bundesverfassungsgerichtes im Juli 1998 beruhigte sich jedoch die Situation und das Medieninteresse an diesem Thema nahm schlagartig ab. Veröffentlichungen in der Zeitschrift "Sprachreport" werden vermehrt öffentlich wahrgenommen. Unter anderem wurden Artikel über verständliche Gebrauchsanweisungen, Probleme mit Anrufbeantwortern, eine Blütenlese der Wahlprogramme zur Bundestagswahl und ein Briefwechsel mit der deutschen Telekom von zahlreichen Rundfunk- und Printmedien aufgegriffen und weiterverbreitet. Erneut fanden in den Räumen des IDS zwei Kunstausstellungen statt, die erste trug den Titel „Begegnung" und zeigte Fotos von Branko Brandays, die zweite mit dem Titel „Überschneidungen" zeigte Arbeiten dreier Künstler aus Österreich, Tschechien und der Slowakei.

Im Juli wurde mit der Überarbeitung des 1994 erschienen Handbuchs zur „Förderung der sprachlichen Kultur in der Bundesrepublik Deutschland" begonnen. In diesem Handbuch soll ein Überblick über Institutionen gegeben werden, die (im weitesten Sinn) für die Förderung der sprachlichen Kultur in Deutschland tätig sind. Das Projekt wird von der Robert-Bosch-Stiftung finanziert und in Zusammenarbeit mit der Gesellschaft für deutsche Sprache durchgeführt (Verantwortlich im IDS: Annette Trabold).

Die „Dokumentation zur Germanistischen Sprachwissenschaft: Sprachwissenschaftliche Forschungsvorhaben" ist als abfragbare Datenbank im WWW veröffentlicht (http://www.ids-mannheim.de/oea/forsch/). Aktualisiert wird diese Datenbank regelmäßig. Rund 700 Projekte sind zum Jahresende verzeichnet.

Das Informationsangebot im Internet über das IDS ist auf 700 Seiten angewachsen. Monatlich können im Durchschnitt ca. 80.000 Zugriffe aus dem In- und Ausland auf IDS-Seiten gezählt werden. Im August 1998 wurden, bedingt durch das Inkrafttreten der Rechtschreibreform, $120.000 \mathrm{Zu}$ griffe registriert. In Zusammenarbeit mit der Arbeitsstelle Linguistische Datenverarbeitung wird das Informationsangebot des IDS im WWW weiter ausgebaut und stetig aktualisiert. Aktuelle Informationen über das IDS können unter http://www.ids-mannheim.de abgerufen werden

In der Bibliothek werden mit dem Katalogisierungssystem BISLOK sämtliche Neuerwerbungen und - soweit möglich - Altbestände erfasst und verschlagwortet.

Die weiterhin zunehmende Zahl von Gastwissenschaftlern und die an- 
wachsende Zahl von Nutzern aus den umliegenden Hochschulen erfordern umfangreiche Beratungstätigkeiten durch die Bibliotheksangestellten. Die von der Bibliothek herausgegebenen Neuerwerbungslisten (Erscheinungsweise 3-4 mal jährlich) dienen der in- und ausländischen Hochschulgermanistik als wichtige Informationsquelle. Sie werden auf Anforderung verschickt und sind darüber hinaus monatlich im Internet aktualisiert abrufbar über http://www.ids-mannheim.de/oea/neueingang.html.

Mitarbeiter der Arbeitsstelle:

Leiterin:

Dr. Annette Trabold, M. A.

Wissenschaftliche Mitarbeiter:

Franz Josef Berens - Monika Kolvenbach, M. A.

Dokumentarin: Dipl.-Dok. Katrin Freese, M. A.

EDV-Mitarbeiter: Claus Hoffmann

Erstellung von Druckvorlagen: Ursula Blum - Cornelia Kayser - Norbert Volz, M. A.

Bibliothek: Lucia Berst-Birgit Günther-Dipl.-Bibl. Eva Teubert(Leitung)

Sekretärin: Barbara Stolz, M.A.

\subsection{Verwaltung und Vorstandssekretariat}

Verwaltungsleiter:

Harald Forschner

Verwaltungsangestellte:

Monika Buchmüller (beurlaubt) - Jean Christoph Clade - Gerhard Köck

- Hildegard Magis - Gerd Piroth - Hermann Schmitt

Telefonzentrale/Poststelle:

Franz-Albert Werner - Hannelore Wittmann

Hausmeister: Uwe Zipf

Vorstandssekretariat: Cornelia Pfützer-König

\section{Tagungen, Kolloquien und Vorträge externer Wissenschaftler am IDS}

3.1 Erste Schritte aus dem Elfenbeinturm Sprachwissenschaftler zeigen sich auf der 34. Jahrestagung des IDS offen für neue Impulse

Die Sprachwissenschaftler scheinen in Bewegung geraten zu sein. Mehr Praxisnähe in Ausbildung und Tätigkeit sowie mehr Interesse für die Belange der Öffentlichkeit - dies waren zentrale Forderungen, die auf der 34. Jahrestagung des Instituts für deutsche Sprache formuliert wurden. Sie sind untrügliche Anzeichen für eine Diskussion, die zwar noch am Anfang steht, gleichwohl Veränderungen erhoffen lässt. So war der Titel der Ta- 
gung „Sprache - Sprachwissenschaft - Öffentlichkeit“" gut gewählt, trug er doch einem innerdisziplinären Trend Rechnung.

Der zaghafte Versuch, den Elfenbeinturm zu verlassen, wird nicht von allen befürwortet. Skeptiker wollen die Festschreibung des Status quo. Die Forschung möge für sich sprechen und die Wissenschaftler sollen nicht nach gesellschaftlicher Anerkennung streben, lässt sich ihr Standpunkt umreißen. Ein Vertreter dieser Position: Werner Welzig. Für den Präsidenten der Österreichischen Akademie der Wissenschaften ist es unhaltbar, „dass wir, statt unsere Arbeit zu tun, uns mit dem Nutzen beschäftigen." Sein Vortrag - ein Versuch, die Frage nach dem Nutzen der Geisteswissenschaften mittels des Märchens Rotkäppchen zu beantworten - stieß nicht durchweg auf Zustimmung. Die Aufgaben der Sprachwissenschaft „sind nicht mit Rotkäppchen zu lösen“, so Siegfried Grosse (Bochum).

Eine „Diskothekisierung“" wie Helmut Henne es nannte, also die Hinwendung zu Oberflächlichem, lehnt das Gros der Sprachwissenschaftler ab, dies machten Vorträge und Diskussionsbeiträge deutlich. So stieß der Vorschlag des Verlegers Florian Langenscheidt (München), öffentlichkeitswirksame „Events“ wie Preisverleihungen für Zeitungen oder Redner zu veranstalten, nicht nur auf Begeisterung. Befremdlich mutete manchem seine „Hitliste“" sprachwissenschaftlicher Themen an, die er als verkaufsfähig pries. Jedoch wurde deutlich, dass Erkenntnisse, die im Fach schon lange bekannt sind, den eigenen Kreis bislang kaum verlassen haben. Ein Überdenken gängiger Publikationsgewohnheiten scheint angebracht.

Eine Konzentration auf reine Marketinggedanken zu Lasten von Forschungsinhalten lehnen viele Sprachwissenschaftler ab. So will Ludwig Jäger (Aachen) wohl eine stärkere Stellung der Linguistik in der Öffentlichkeit, verwahrte sich aber gegen den Trend, nur die „Werbung auf dem Markt" zu verbessern. Zu komplex seien die Gründe für die Krise der Linguistik wie der Wissenschaften überhaupt. Jägers Lösungsideen: Die Linguistik muss sich aus ihrer kultur- und medienwissenschaftlichen Isolation lösen und am interdisziplinären Diskurs über eine „Neubestimmung der Geisteswissenschaften als Kulturwissenschaften" beteiligen. Die Linguistik muss ferner neue Themen und Praxisfelder besetzen und die Kommunikation mit der Gesellschaft verbessern. Jäger schwebt hierzu eine „Kultur des Wissenschaftsjournalismus" vor.

Das Verhältnis von Sprache, Sprachwissenschaft und Öffentlichkeit wurde auf der Tagung aus verschiedenen Blickwinkeln beleuchtet. Über die Einstellung der Deutschen zur Sprache referierte Gerhard Stickel (IDS) auf der Basis einer Umfrage in Ost und West. Mehr als die Hälfte der 2000 Befragten zeigte ,,sich sprachlich wenig oder gar nicht interessiert", 43 Prozent hatten ein starkes bis mittleres Interesse. Das Image der deutschen Sprache ist schlecht. Nur knapp fünf Prozent äußerten sich über ihren Zustand positiv, alle anderen negativ. Dialekt hingegen ist in: Zwei 
Drittel der Befragten mögen Sprecher mit regionalem Zungenschlag. Erfreulich und nach Stickel ,überraschend": In Politik und Wirtschaft mag es zwischen Ossis und Wessis kriseln, aber sprachlich klappt es prima. Auch die Mehrsprachigkeit im Land ist für viele kein Problem.

Einen historischen Blickwinkel nahm Helmut Henne (Braunschweig) ein. Er stellte die großen Epochen und ihre bekanntesten Vertreter vor: die reflexive Sprachlehre von Adelung, die historische Sprachwissenschaft mit politischen Akzenten von Grimm und den Junggrammatiker Paul. Er zog das Fazit, dass die Sprachwissenschaft ihre eigene Geschichte aufarbeiten müsse, um aus Fehlern der Vergangenheit zu lernen.

Das aktuelle Verhältnis von Linguistik und Öffentlichkeit beleuchtete Gerd Antos (Halle-Wittenberg) anhand einer Umfrage unter etablierten Wissenschaftlern. Die vielen emotionalen Kommentare der Befragten lassen laut Antos den Schluss zu, dass es sich um ein ,neuralgisches“ Thema handelt. Viele beklagen, dass sprachwissenschaftliche Themen öffentlich diskutiert werden, aber die Sprachwissenschaftler nicht gehört werden. Diese Klage wurde auch auf der Tagung mehrfach geäußert, u. a. von Rudolf Hoberg (Darmstadt) während der Podiumsdiskussion. Ein erklärtes Ziel der begonnenen Diskussion ist deshalb: Die Gesellschaft soll Interesse an den Sprachwissenschaftlern und ihrem Wissen über die Sprache gewinnen.

Peter Eisenberg (Potsdam) zeigte u. a. am Beispiel sprachkritischer ÄuBerungen des Journalisten Dieter E. Zimmer, wie grammatikalische Kenntnisse für den öffentlichen Diskurs über Sprache nutzbar gemacht werden können. Er verdeutlichte, dass Kritik an sprachlichen Entwicklungen oft auf fehlenden Kenntnissen von Grammatikregeln beruht.

Die in den Medien geführte Diskussion über die Rechtschreibreform zeichnete Werner Eroms (Passau) nach. Seine Analyse von über $2000 \mathrm{Be}-$ richten, Interviews, Kommentaren und Leserbriefen machte deutlich, dass die Journalisten sich viel neutraler über die Reform geäußert haben als die Leserbriefschreiber. Dort standen nicht Sachthemen, sondern Glaubensbekenntnisse im Vordergrund: die eigene Sprachauffassung und die Verdammung der Andersdenkenden. Die Analyse veranlasste Eroms, Kritik an der eigenen Zunft zu äußern: Die Linguisten hätten zu sehr politisch statt sprachwissenschaftlich argumentiert, und die öffentliche Auseinandersetzung habe dem Image des Faches geschadet. Dies unterstrich auch Fritz Kuhn (MdL) in seiner Wissenschaftlerschelte während der Podiumsdiskussion.

Ein gelungenes Beispiel für den Einfluss der Sprachwissenschaft auf die Gesellschaft stellte Gisela Schoenthal (Freiburg) vor. Die feministische Sprachkritik hat „einen Sprachwandel von unten ausgelöst" und zu Veränderungen in vielen Bereichen geführt. Institutionalisiert wurden weibliche Bezeichnungen in Vorgaben für die Verwaltungs- und Gesetzes- 
sprache. Die feministische Sprachkritik inspirierte Autoren literarischer Werke - als Beispiel sei Günter Grass' Rättin genannt - und beeinflusste den allgemeinen Sprachgebrauch. Je nach Textsorte und Kontext finden sich heute movierte Formen (v. a. bei Berufsbezeichnungen), das Binnen-I (StudentInnen), Paarformen (Teilnehmerinnen und Teilnehmer) oder neutralisierende Bezeichnungen (Studierende).

Die Öffentlichkeit wurde nicht nur thematisiert, sondern kam auf der Tagung auch selbst zu Wort; zwar nicht der Mann oder die Frau von der Straße, wohl aber Vertreter verschiedener Berufe. Sie äußerten vor allem ihre Wünsche an die Sprachwissenschaft.

Für die Berufsgruppe der Journalisten sprach Rudolf Gerhardt (Mainz). Der promovierte Jurist und frühere FAZ-Mitarbeiter, der in der Journalistenausbildung tätig ist, forderte die Sprachwissenschaftler auf, den Journalisten kritisch auf die Finger zu schauen. Am Beispiel von Euphemismen, falschen Sprachbildern und manipulativen Wortschöpfungen, die man in Zeitung, Rundfunk und Fernsehen täglich antrifft und über die Medien Einlass in den Sprachgebrauch finden, machte er diese Notwendigkeit deutlich.

Cathrine Fabricius-Hansen (Oslo) formulierte die Anliegen der Auslandsgermanisten, deren Tätigkeit vor allem durch die Vermittlung sprachlicher Kenntnisse an Nicht-Muttersprachler geprägt ist. Ihre Wünsche: einfache Einführungen in Grundkenntnisse des Faches, Monographien zu sprachwissenschaftlichen Schwerpunkten, Beschreibungen des Sprachgebrauchs und eine Übersicht über die Dialekte inklusive „Kostproben“ auf CD. Ferner wünschte sie sich ein stärkeres Interesse an didaktischen Fragen. Mit diesem Wunsch schien sie bei vielen offene Türen einzulaufen. Eva Neuland (Wuppertal) unterstrich mehrfach, dass Linguistik und Didaktik stärker verbunden werden sollten. Für sie sind Schüler Multiplikatoren sprachwissenschaftlicher Erkenntnisse, eine Überzeugung, die sie mit anderen teilte, z. B. mit Gerhard Voigt-Schneekloth (Berlin).

Er beklagte in seinem Vortrag die fehlende sprachwissenschaftliche Kompetenz seiner Kolleginnen und Kollegen im Schuldienst. Sein Lösungsvorschlag, um die Linguistik mehr in die Schulen zu bringen: Germanistikstudierende müssten stärker für die Sprachwissenschaft gewonnen werden. Nicht ganz so schwarz wie vom Referenten gezeichnet, sahen viele Diskussionsteilnehmer die Situation. Sie unterstrichen, dass die Lehrer jüngeren Datums weit mehr Interesse am Fach zeigten als ihre Vorgänger.

Beispiele für anwendungsbezogene Tätigkeiten gibt es bereits: Gisela Brünner (Dortmund) und Michael Becker-Mrotzek (Münster) arbeiten seit Jahren im Bereich der Angewandten Gesprächsforschung. Als Kommunikationstrainer sind sie für Wirtschaft, Verwaltung, Schulen und andere Institutionen tätig. Keine reinen Analysen, sondern wissenschaftlich fundierte Evaluation der Gespräche wünschen die Praktiker; ein Anspruch, 
der nach Überzeugung der Referenten der Sprachwissenschaft neue Impulse gibt.

Matthias Jung und Martin Wengeler (Düsseldorf) berücksichtigen gesellschaftliche Themen in ihrer Forschung. Ihr Ansatz ermöglicht es, ,diskursbegleitend" öffentliche Diskussionen sprachwissenschaftlich zu analysieren. Dabei untersuchen sie nicht isolierte Wörter, sondern Lexeme, Metaphern und Argumentationsmuster, die in einem Diskurs - etwa über Ausländer - zentral sind, unter Berücksichtung des Kontextes und ihrer Funktionen. Trotz aller Probleme der Disziplin, über die eigenen Grenzen hinaus zu wirken, sehen sie die Entwicklung der Sprachwissenschaft positiv: „Und sie bewegt sich doch“, lautet ihre Situationsbeschreibung.

Beispiele für erste Veränderungen nannten auch Josef Klein (Koblenz), der eine Studie über die Rezeption von wirtschaftlichen Fachbegriffen jüngst beendet hat, sowie Theo Bungarten (Hamburg), der mit dem dortigen Arbeitskreis „Unternehmenskommunikation“ schon vielfach für die Wirtschaft tätig war. Gute Chancen misst Achim Struchholz, PR-Mann aus Düsseldorf, einer Zusammenarbeit zwischen Wirtschaft und Linguistik bei.

Das eine machen und das andere nicht lassen. Dies scheint der gemeinsame Nenner zu sein, auf den sich die meisten Tagungsteilnehmerinnen und -teilnehmer einigen zu können scheinen. Will heißen: Mehr Öffnung nach außen, mehr verständliche Texte für Laien, aber nicht auf Kosten der Inhalte oder grundlegender genuin sprachwissenschaftlicher Forschung. In Zeiten leerer Kassen und eines härteren Verteilungskampfes zwischen den Wissenschaften scheint eine Außenprofilierung unerlässlich. In diese Richtung wies der Redebeitrag von Annette Trabold. Die Pressesprecherin des IDS unterstrich ferner, dass eine politische Lobby geschaffen werden müsse, ,damit Grundlagenforschung weiterhin möglich ist“.

Dass das Institut für deutsche Sprache auch weiterhin Diskussionen anregen will, die aus dem Zentrum der eigenen Disziplin hinausreichen, macht das Thema der kommenden Jahrestagung deutlich. Über ,Sprache und (neue) Medien" sollen 1999 Sprachwissenschaftlerinnen und Sprachwissenschaftler in Mannheim diskutieren.

\subsection{Symposium „Computergestützte Produktion und Publikation von Wörterbüchern"}

Am 23.9. - 25.9.1998 fanden sich an der Heidelberger Akademie der Wissenschaften über 100 Interessierte aus Wissenschaft, Verlagen und Industrie zum Symposium „Computergestützte Produktion und Publikation von Wörterbüchern" zusammen. Diskutiert wurden die Perspektiven, die sich durch Neue Medien für die Lexikographie eröffnen; in den Foren „Werkzeuge" und „Projekte" trafen sich Softwareanbieter mit Anwendern aus lexikographischen Projekten. Das Symposium wurde gemeinsam ver- 
anstaltet von der Heidelberger Akademie der Wissenschaften, dem IDS und dem Arbeitskreis „Hypermedia“ der Gesellschaft für linguistische Datenverarbeitung GLDV; organisiert und geleitet wurde die Veranstaltung von Ingrid Lemberg, Bernhard Schröder und Angelika Storrer.

\subsection{Vorträge externer Wissenschaftler im IDS 1998}

17.2.1998 Prof. Dr. Hans-Jürgen Bucher (Universität Trier), „Vom Textdesign zum Hypertext. Probleme der Wissensvermittlung in Print- und Online-Medien“

14.9.1998 Prof. Yutaka Wakisaka (Tenri-Universität Nara/Japan), „Metaphern und Idiome. Überlegungen zu kommunikativen und rhetorischen Funktionen"

5.10.1998 PD Dr. Ulrike Haß-Zumkehr/PD Dr. Claudia Fraas (beide IDS) und Dr. Rolf Assfalg (Universität Konstanz), „Infos und Vorführung über das neue Projekt der Abteilung Lexik"

4.12.1998 Prof. Kusshid Ahmad (Universität Surrey), „Corpus Linguistics and the "End of Text"

\section{Lehraufträge und Vorträge von IDS-Mitarbeitern außerhalb des Instituts}

\subsection{Lehraufträge}

\section{Prof. Dr. Joachim Ballweg}

SS 1998, Einführung in die Sprachwissenschaft, Proseminar, Universität Karlsruhe

SS 1998, Semantik, Hauptseminar, Universität Karlsruhe

Dr. Birgit Barden

WS 1998/99, Sprache und Identität, Proseminar, Universität Mainz/ Germersheim

Dr. Eva Breindl

WS 1997/98 (zusammen mit Dr. Renate Pasch) Deutsche Konnektoren, Hauptseminar, Universität Heidelberg

SS 1998, Deutsch als Fremdsprache und neue Medien, Seminar, Universität Mainz

Dr. Elke Donalies

WS 1998/99, Die Wortbildung im Deutschen, Seminar, Universität Heidelberg

Prof. Dr. Reinhard Fiehler

SS 1998, Sprache und Kommunikation im Alter, Blockseminar, Universität Bielefeld

WS 1998/99, Verständigungsprobleme in betrieblicher Kommunikation, Blockseminar, Universität Wien/Österreich 
PD Dr. Claudia Fraas

SS 1998, Kognition und Kommunikation - Oder: Die Archäologie des Wissens, Hauptseminar, Universität Mannheim

WS 1998, Sprachliche Kommunikation - Kultur, Strategien, Konflikte, Hauptseminar, Universität Mannheim

Prof. Dr. Gisela Harras

SS 1998, Wieviel Grammatik braucht der Mensch/die Frau/der/die Germanist/in? Hauptseminar, Universität Mannheim

WS 1998/99, Wahl(ver)sprechen - Zum Sprachverhalten im Bundestagswahlkampf 1998, Hauptseminar, Universität Mannheim

PD Dr. Ulrike Haß-Zumkehr

SS 1998, Klassische Texte zur Semantik, Proseminar, Universität Heidelberg

WS 1998/99, Sprache und Nation. Zur Geschichte zweier Leitideen in der deutschen Sprach-, Wissenschafts- und Gesellschaftsgeschichte, Vorlesung, Universität Mannheim

\section{Prof. Dr. Werner Kallmeyer}

SS 1998 (zusammen mit Prof. Dr. Christine Bierbach) Pragmatik und Soziolinguistik, wissenschaftliches Kolloquium, Universität Mannheim SS 1998, Formulierungsverfahren in mündlicher Kommunikation, Hauptseminar, Universität Mannheim

WS 1998/99, Formulierungsverfahren und rhetorische Strategien, Hauptseminar, Universität Mannheim

WS 1998/99 (zusammen mit Prof. Dr. Christine Bierbach) Pragmatik und Soziolinguistik, wissenschaftliches Kolloquium, Universität Mannheim

PD Dr. Inken Keim

SS 1998/WS 1998/99, Pidgin und Kreolsprachen, Hauptseminar, Universität Mannheim

WS 1998/99, Perspektivierung in Gesprächen, Hauptseminar, Universität Saarbrücken

PD Dr. Katharina Meng

SS 1998, Migration und Zweisprachigkeit, Seminar, PH Heidelberg

Prof. Dr. Wolfgang Motsch

SS 1998, Überblick über die deutsche Wortbildung, Vorlesung, JozsefAttila-Universität Szeged/Ungarn

SS 1998, Übungen zur deutschen Wortbildung, Seminar, Jozsef-AttilaUniversität Szeged/Ungarn

SS 1998, Die sprachliche Form von Sprechakten, Seminar, Jozsef-Attila-Universität Szeged/Ungarn 
Pantelis Nikitopoulos

SS 1998, Interkulturelle Erziehung und Binnendifferenzierung im Unterricht der Regelklasse, Seminar, PH Heidelberg

Dr. Renate Pasch

WS 1997/98 (zusammen mit Dr. Eva Breindl) Deutsche Konnektoren, Hauptseminar, Universität Heidelberg

Dr. Rudolf Schmidt

WS 1998/99, Digitale Sprach- und Bildverarbeitung, Vorlesung, Berufsakademie Mannheim

PD Dr. Thomas Spranz-Fogasy

SS 1998, Sprache und Situation, Hauptseminar, Universität Mannheim

Prof. Dr. Gerhard Stickel

WS 1998/99, Sprachkritik, Sprachplanung und Sprachpflege in Deutschland, Hauptseminar, Universität Mannheim

Dr. Angelika Storrer

SS 1998, Textbegriff und Neue Medien, Hauptseminar, Universität des Saarlandes

WS 1998/99 (zusammen mit Prof. Dr. Dr. h.c. Herbert Ernst Wiegand), Lexikographie und Neue Medien, Forschungsseminar, Universität Heidelberg

Prof. Dr. Bruno Strecker

WS 1998/99, Syntax und Semantik nicht-propositionaler Ausdruckseinheiten im Deutschen, Hauptseminar, Universität Augsburg

Dr. Peter Wagener

SS 1998, Einführung in die Allgemeine Linguistik, Proseminar, Universität Mannheim

9.-23.5.1998, Dialektologie des Deutschen, Vorlesung und Hauptseminar (Kompaktveranstaltungen, TEMPUS-Programm, EU), Universität Sarajevo

Prof. Dr. Gisela Zifonun

WS 1998/99, Syntax, Vorlesung, Universität Mannheim

\subsection{Kurse und Kurzseminare}

Prof. Dr. Joachim Ballweg

26.11.1998, Semantik der deutschen Determinative, Workshop, IDS, Mannheim 


\section{Cyril Belica}

10.3.1998, Workshop „COSMAS I im World Wide Web“, Vortrag und Vorführung, 34. Jahrestagung des IDS, Mannheim

11.3.1998, Workshop „Morphosyntaktische Annotationen in COSMAS

I“, Vortrag und Vorführung, 34. Jahrestagung des IDS, Mannheim

Dr. Ursula Brauße

23.10.1998, Co-presentation: Adversative connectors on distinct levels (Ewald Lang), Workshop „Cognitive and Discourse Perspectives on Cause, Condition, Concession and Contrast", Universität Konstanz

Dr. Eva Breindl

15.7.1998, Deutsch lernen und lehren mit neuen Medien, Lehrerfortbildungsseminar für ausländische Deutschlehrer, Goethe-Institut München 3.8.1998, Workshop „Das Internet als Referenzquelle zur deutschen Sprache: Übersicht, Evaluation, Bedarf", Fortbildungsseminar für DAAD-Lektorinnen „Neue Medien und DaF“, DAAD, Bonn

Prof. Dr. Gisela Harras

5.5.1998, Lokalisierungsanweisungen, Sonderforschungsbereich „Situierte künstliche Kommunikatoren“, Universität Bielefeld

PD Dr. Ulrike Haß-Zumkehr

26.8.1998, Sprachbildung gestern und heute. Zum Daniel-SandersSprachpreis für Schülerinnen und Schüler, Fortbildungsveranstaltung für Lehrer des Landkreises Mecklenburg-Strelitz, Alt-Strelitz

Dr. Klaus Heller

9.11.1998, Verschiedene Aspekte von Norm und Variabilität in der Schreibung und insbesondere zur Neuregelung der deutschen Rechtschreibung und zu deren Hintergründen, Seminar, Fraunhofer-Gesellschaft, München

Prof. Dr. Werner Kallmeyer

16.10.-17.10.1998, Analyse des politischen Diskurses zur Europapolitik, Arbeitstreffen, Institut für Angewandte Sprachwissenschaft, Wien

4.12.1998, Sprachbiographien - Erhebungen und Analysen, Universität Basel/Schweiz

Dr. Heidrun Kämper

26./27.10.1998, Erstes Arbeitsgespräch der Projektgruppe „Haltungen zu Europa - Attitudes towards Europe“, University of Durham/England

\section{Pantelis Nikitopoulos}

26.-28.2.1998, Erhöhung der kommunikativen Kompetenz. Möglichkeiten, Inhalte und Techniken der Unterrichtsgestaltung, Workshop, Drei-Länder-Projekt der EU zur Erhöhung der Resozialisierungschancen von Strafgefangenen, Mannheim 
PD Dr. Thomas Spranz-Fogasy

31.1.1998, Ärztliche Fragetechniken, Workshop, Universität Bielefeld

Prof. Dr. Gerhard Stickel

11./12.12.1998, Aktuelle Tendenzen der deutschen Sprache, Goethe-Institut Amsterdam/Niederlande

Dr. Angelika Storrer

5.-9.10.1998, Elektronische Wörterbücher, Blockseminar, Universität Heidelberg

Dr. Peter Wagener

3.6.1998, Zur Rolle der Phonetik im Deutschen Spracharchiv, Kolloquium, IDS, Mannheim

11.9.1998, Zur Gliederung deutscher Dialekte, Kolloquium, Seminar für Sprachmethodik, Burbach

Dr. Edeltraud Winkler

15.5.-16.5.1998 (zusammen mit Prof. Dr. Gisela Harras organisiert), Valenzen, Argumente und Szenen, Workshop, IDS, Mannheim

Dr. Ricarda Wolf

12.-14.2.1998, Gattungsnormen im Bewerbungsgespräch (Koreferat zum Beitrag von Karin Birkner), Workshop: „Fremde im Gespräch“, Universität Hildesheim

\subsection{Vorträge}

Prof. Dr. Joachim Ballweg

9.7.1998, Determinative, Pluralnomina und Substanznomina, Universität Frankfurt

19.9.1998, Iznogud - Kalauer in Comics und das Problem ihrer Übersetzung, Université Paris X (Nanterre)/Frankreich

27.11.1998, Welche Kategorie hat solch-?, Zentrum für allgemeine Sprachwissenschaft, Berlin

Dr. Birgit Barden

3.3.1998, Macht der Ton den Operator? Prosodische Eigenschaften der Operator-Skopus-Struktur, 2. Arbeitstreffen „Linguistische Pragmatik", Halle

1.4.1998, Operator-Skopus-Strukturen und deren prosodische Realisierungen, Neuere Entwicklungen in der Gesprächsforschung, Pragmatisches Kolloquium Freiburg

17.4.1998, Attitudes and social networks and their influence in long term linguistic accommodation, DIS-UNIFICATION, Competing Con- 
structions of Contemporary Germany, University of Southampton/Großbritannien

27.11.1998, Sprachliche Akkommodation und soziale Integration, Workshop des Fachbereichs Germanistik, FU Berlin

Dr. Karl-Heinz Bausch

19.10.1998, Dialektologie und interaktionale Soziolinguistik (am Beispiel des Sprachwandels im Pfälzischen), Internationale Dialektologentagung „Dialektologie zwischen Tradition und Neuansätzen“, Göttingen

\section{Franck Bodmer}

6.2.1998 (zusammen mit Dr. Wilfried Schütte), SERGES: Acquisition and Maintenance of Spoken Language, Workshop: Constructing and Accessing Multi-Media Corpora, Max-Planck-Institut für Psycholinguistik, Nijmegen/Niederlande

18.2.1998, An overview of COSMAS II, Institut für Wirtschaftsinformatik, Universität Kolding/Dänemark

7.-8.5.1998, Cosmas II Progress Report, Meeting of the Academic Development Group of COSMAS II, Norwegian Center for the Computing in the Humanities, Universität Bergen/Norwegen

Dr. Eva Breindl

10.2.1998, Das Handbuch der deutschen Konnektoren als Hypertext, HU Berlin

2.7.1998, „Mehrwert Internet“ oder doch nur „Des Kaisers neue Kleider"?, GoeDaF-Kolloquium (Goethe-Institut und Institut für Deutsch als Fremdsprache der Universität München), Goethe-Institut München 3.8.1998, Deutsch als Fremdsprache übers Internet: Mehrwert oder Rückschritt?, Einleitungsvortrag zu einem DAAD-Lektoren-Fortbildungsseminar „DaF und Neue Medien“, DAAD, Bonn

Prof. Dr. Reinhard Fiehler

6.2.1998, Interaktive Darstellung von Alter im Gespräch, Universität Bonn

3.3.1998, Operator-Skopus-Strukturen in gesprochener Sprache, 2. Arbeitstreffen „Linguistische Pragmatik“, Halle

28.4.1998, Sprachliche Varianz im Alter, Wissenschaftliches Kolloquium, Universität Mannheim

28.9.1998, Kommunikationsberatung und -training auf gesprächsanalytischer Basis, IHK-Kolloquium „Sprache - Erfolgsfaktor in der Wirtschaft", IDS, Mannheim

15.12.1998, Kommunikation im Alter, Wiener Sprachgesellschaft, Wien/Österreich 
PD Dr. Claudia Fraas

27.5.1998, Karrieren geschichtlicher Grundbegriffe - FREIHEIT, GLEICHHEIT, BRÜDERLICHKEIT, Ringvorlesung, Universität Mannheim

5.10.1998 (zusammen mit PD Dr. Ulrike Haß-Zumkehr und Dr. Rolf Assfalg) Infos und Vorführung zum Projekt LEKSIS, Kolloquium, IDS, Mannheim

Prof. Dr. Gisela Harras

4.5.1998, Tendenzen der Lexikalisierung kommunikativer Konzepte, Universität Bielefeld

25.6.1998, Lokalisierungsverben, Lexikographisches Kolloquium, Universität Heidelberg

21.9.1998, Sprache als Lebenswelt, „Mathematik und Lebenswelt“", TU Darmstadt

29.10.1998, Lexikologie und Lexikographie - kein Verhältnis? Linguistisches Kolloquium, Universität Jena

PD Dr. Ulrike Haß-Zumkehr

29.1.1998, Spiegelungen der Rechtssprache in der Lexikografie, Internationale Fachtagung „Recht und Sprache in der deutschen Aufklärung", Heidelberg

26.8.1998, Daniel Sanders und seine Auseinandersetzung mit den Brüdern Grimm, Hans-Fallada-Klub Neustrelitz

24.9.1998, Projekt: LEKSIS - Wissen über Wörter, Symposium „Computergestützte Produktion und Publikation von Wörterbüchern", Heidelberg

25.9.1998, Zukunft der Wörterbücher - Wörterbücher der Zukunft, Statement, Podiumsdiskussion, Symposium „Computerunterstützte Produktion und Publikation von Wörterbüchern", Akademie der Wissenschaften, Heidelberg

5.10.1998 (zusammen mit PD Dr. Claudia Fraas und Dr. Rolf Assfalg) Infos und Vorführung zum Projekt LEKSIS, Kolloquium, IDS, Mannheim

Dr. Klaus Heller

Unter verschiedenen Aspekten wurden Vorträge zu Norm und Variabilität in der Schreibung und insbesondere zur Neuregelung der deutschen Rechtschreibung und zu deren Hintergründen gehalten:

20.1.1998, IDS, Mannheim

16.2.1998, IDS, Mannheim

4.5.1998, Gesellschaft für deutsche Sprache, Halle

5.5.1998, Institut für deutsche Sprache und Kultur, Universität HalleWittenberg, Wittenberg-Kolleg/Leucorea, Wittenberg 
14.10.1998, Lutzenbergschule Mannheim

3.11.1998, Kölner Journalistenschule für Politik und Wirtschaft, Köln

7.11.1998, Förderkreis der Schriftsteller in Sachsen-Anhalt, Großjena

9.11.1998, Fraunhofer-Gesellschaft, München

28.11.1998, Høgskolen i Agder, Nordisk Språknormeringskonferanse, Kristiansand/Norwegen

Dr. Manfred Hellmann

24.6.1998, Sprache und Kommunikation in Deutschland nach der Wende und ihre Untersuchung im IDS, IDS, Mannheim

26.6.1998, Sprache und Kommunikation in Deutschland seit der Wende, IDS, Mannheim

Prof. Dr. Dieter Herberg

26.10.1998, Schlüsselwörter. Sprache und Politik zur Zeit der deutschen Wiedervereinigung, Projekt „Haltungen zu Europa - Attitudes Towards Europe“, University of Durham/England

Prof. Dr. Werner Kallmeyer

3.2.1998 (zusammen mit Prof. Dr. Christine Bierbach/PD Dr. Inken Keim), Kommunikativer sozialer Stil von jugendlichen Migrantengruppen in Mannheim, Wissenschaftliches Kolloquium, Universität Mannheim

3.4.1998, Sprachliche Verfahren der sozialen Integration und Ausgrenzung, Universität Dortmund

14.5.1998, Advances in Sociolinguistics, Jahreskongress des finnischen Linguistenverbandes, Universität Tampere/Finnland

14.6.1998 (zusammen mit Prof. Dr. Christine Bierbach/PD Dr. Inken Keim), Sprachvariation in Jugendgruppen der zweiten Migrantengeneration, Wissenschaftliches Kolloquium, Universität Mannheim

1.7.1998, Kommunikation vor Gericht, Deutsche Richterakademie, Tagung „Recht und Sprache“, Trier

23.7.1998, Others' inserts in an ongoing turn. Some sequential, grammatical and rhetorical observations, 6 . Kongress der Internationalen Gesellschaft für Pragmatik, Reims/Frankreich

24.7.1998 (zusammen mit PD Dr. Inken Keim), Linguistic variation and the construction of social identity in a German Turkish setting, IPRA, Reims/Frankreich

23.11.1998, Sprachvariation und soziale Stilistik, Kolloquium „Vom Umgang mit Variation: Wissenschaftsgeschichte, Dialektologie, Soziolinguistik", Kolloquium zum 60. Geburtstag von Heinrich Löffler, Universität Basel

15.12.1998, Perspektivität und Perspektivierung im Diskurs, Universität Essen 
Dr. Heidrun Kämper

3.4.1998, Schlagwörter der Revolution von 1848 bei Hoffmann von Fallersleben, Hoffmann von Fallersleben-Symposion, Hoffmann von Fallersleben-Gesellschaft/Seminar für deutsche Sprache und Literatur der TU Braunschweig, Wolfsburg-Fallersleben

3.7.1998, Er war immer hilfsbereit - Entlastungsargumente und Sprachgeschichte der frühen Nachkriegszeit, TU Braunschweig

\section{PD Dr. Inken Keim}

3.2.1998 (zusammen mit Prof. Dr. Christine Bierbach/Prof. Dr. Werner Kallmeyer), Kommunikativer sozialer Stil von jugendlichen Migrantengruppen in Mannheim, Wissenschaftliches Kolloquium, Universität Mannheim

5.3.1998, Soziale und kulturelle Selbstdefinition in einer Jugendgruppe ausländischer Herkunft: Die interaktive Konstitution der Kategorie „Migrant/Migrantin“, 20. Jahrestagung der DGfS, Universität Halle 14.6.1998 (zusammen mit Prof. Dr. Christine Bierbach/Prof. Dr. Werner Kallmeyer), Sprachvariation in Jugendgruppen der zweiten Migrantengeneration, Wissenschaftliches Kolloquium, Universität Mannheim 24.7.1998 (zusammen mit Prof. Dr. Werner Kallmeyer), Linguistic variation and the construction of social identity in a German Turkish setting, IPRA, Reims/Frankreich

Jacqueline Kubczak

16.5.1998, Nachdenken über „verletzen“" und die Folgen, Workshop: Valenzen, Argumente und Szenen, IDS, Mannheim

Dr. Ann Lawson

3.4.1998 (zusammen mit Eric Windisch), Corpus-based Dictionaries of Collocation - Comparing English and German Words in Use, EURALEX 3rd International Symposium on Phraseology, Stuttgart 8.5.1998, Capturing Collocation: word combinations in dictionaries, "Methodological Questions in Corpus Linguistics“, Perpignan/Frankreich

28.5.1998, East meets West: Producing Multilingual Resources in a European Context (Session Natural Language Corpus), LREC First International Conference on Language Resources and Evaluation, Grana$\mathrm{da} /$ Spanien

5.6.1998, Compiling a Parallel Corpus: Issues from Personal Experience, Universidad Europea de Madrid, Departamento de Traduccion e Interpretacion, Madrid/Spanien

19.6.1998, The Bridge Dictionary Multilingual Database, PAROLE/Tuscan Word Centre Workshop on Multilingual Lexical Semantics, Pescia/Italien 
PD Dr. Katharina Meng

15.1.1998, Biographisches Erzählen, 6. Workshop „Sprachforschung, Sprachlehre", Universität Hamburg

27.1.1998, Code-Switching deutsch-russisch, Soziolinguistisches Kolloquium, Universität Mannheim

5.3.1998, Deutsch-russisch gemischtsprachige Äußerungen und ihre Wahrnehmung durch russlanddeutsche Sprecher, 20. Jahrestagung der DGfS, Arbeitsgruppe ,,Sprachliche Kreationen in der Migrationsgesellschaft", Universität Halle

1.12.1998, Bedingungen und Verläufe der sprachlichen Integration von Russlanddeutschen, Universität Dortmund

Dr. Wolfgang Mentrup

12.5.1998, Gibt es in der deutschen Sprachgemeinschaft eine „allgemein anerkannte" Rechtschreibung? Mündliche Verhandlung zur Verfassungsbeschwerde gegenüber der Einführung der sogenannten Rechtschreibreform, Bundesverfassungsgericht Karlsruhe

28.11.1998, Über Einheit und Variation in der Sprachnorm - Historische Rückblicke, ausgehend von der neuen deutschen Rechtschreibung, Nordisk språknormeringskonferanse: Der Sprachbenutzer und die Wahlfreiheit, Homogene oder heterogene Norm? Institutt for nordisk og mediefag, Kristiansand/Norwegen

Prof. Dr. Wolfgang Motsch

28.04.1998, Wie regelmäßig sind Wortbildungsregeln? Sprachwissenschaftliche Sektion der Filiale der Ungarischen Akademie der Wissenschaften in Szeged/Ungarn

08.05.1998, Theoretische Grundlagen der Wortbildung, Institut für Sprachwissenschaft der Ungarischen Akademie der Wissenschaften, Budapest/Ungarn

Dr. Renate Pasch

23.10.98, German wo - both causal and concessive? Workshop on "Cognitive and Discourse Perspectives on Cause, Condition, Concession and Contrast“", Universität Konstanz

Prof. Dr. Hartmut Schmidt

5.6.1998, Zur Einführung elektronischer Datenbanken in eine korpusbasierte Wortschatzforschung, Kommission fuir Literarische $\mathrm{Ge}$ brauchsformen der Österreichischen Akademie der Wissenschaften, Wien

Dr. Reinhold Schmitt

19.05.1998, Schnupperkurs „Gesprächsanalyse“, SAP-Walldorf 28.09.1998, Sprache - Erfolgsfaktor in der Wirtschaft, IHK-Kolloquium ,Sprache - Erfolgsfaktor in der Wirtschaft“, IDS, Mannheim 
Dr. Wilfried Schütte

6.2.1998 (mit Franck Bodmer), SERGES - Acquisition and Maintenance of Spoken Language, Workshop: Constructing and Accessing MultiMedia Corpora, Max-Planck-Institut für Psycholinguistik, Nijmegen/Niederlande

16.7.1998, Text-Ton-Alignment von Korpora gesprochener Sprache, Kolloquium, IDS, Mannheim

17.10.1998, Formen der Kommunikationsregulierung in Fernsehgesprächen: Die autoreflexive Talkshow, Arbeitstreffen, Zentrum für internationale und interdisziplinäre Studien, Wien/Österreich

PD Dr. Thomas Spranz-Fogasy

30.1.1998, Die Rolle des Gesprächs im Zusammenhang mit medizinischer Diagnostik und Therapie, Podiumsdiskussion, Universität Bielefeld

1.5.1998, David und Goliath - Kommunikative Strategien von Bürgern in umweltpolitischen Auseinandersetzungen mit Behörden und Verwaltung, Energiewirtschaftliches Gespräch der Energieversorgung WeserEms AG, Juist

18.6.1998, Interactional Resources of Argumentation. 4th International Conference on Argumentation. International Society for the Study of Argumentation, Amsterdam/Niederlande

28.9.1998, Interkulturelles Verhandeln, IHK-Kolloquium „Sprache Erfolgsfaktor in der Wirtschaft", IDS, Mannheim

Dr. Kathrin Steyer

28.4.1998, The corpus-based electronic dictionary of German-FrenchCollocations - aims, methods, tools and questions. Report on a new collocation dictionary project of the Institute for the German Language (IDS) Mannheim, InterLex98, University of Exeter/Großbritannien 7.6.1998 (zusammen mit Prof. Josef Klein), Von der Fraktionssitzung zum Pressekommentar - Parlamentarischer Diskurs in der Mediengesellschaft, „Sprache des deutschen Parlamentarismus“, Arbeitstagung der AG „Sprache in der Politik“ e. V., Magdeburg

Prof. Dr. Gerhard Stickel

10.3.1998, Zur Sprachbefindlichkeit der Deutschen: Erste Ergebnisse einer Repräsentativumfrage, 34. Jahrestagung des IDS, Mannheim 1.4.1998, Entwicklungstendenzen der deutschen Gegenwartssprache, Goethe-Institut Tokio/Japan (auch am 9.4.1998 an der Universität Kagoshima/Japan, am 11.4.1998 an der Kyushu-Universität, Fukuoka/Japan)

6.4.1998, Der Streit um die Reform der deutschen Rechtschreibung, Goethe-Institut Osaka/Japan 
14.7.1998, Was halten die Deutschen von ihrer Sprache?, Universität Göttingen

18.11.1998, Leibniz und die deutsche Sprache, Jahrestagung der Wissenschaftsgemeinschaft Gottfried Wilhelm Leibniz, Berlin

3.12.1998, Hochdeutsch als normativer Begriff, Institut für Deutsch als Fremdsprache, Universität München

\section{Dr. Angelika Storrer}

5.5.1998, Hypertext: Kohärenzbildung und Navigationsmittel, Universität Zürich

15.5.1998, Valenz und Empirie, Mannheim

24.9.1998, Hypermedia in der Lexikographie, Symposium „Computergestützte Produktion und Publikation von Wörterbüchern", Akademie der Wissenschaften, Heidelberg

25.9.1998, Sprachtechnologie für die Lexikographie, Symposium "Computergestützte Produktion und Publikation von Wörterbüchern“, Akademie der Wissenschaften, Heidelberg 10.11.1998, Schriftverkehr auf der Datenautobahn: Besonderheiten der schriftlichen Kommunikation im Internet, Ringvorlesung „Sprache Schrift - Schreiben", Universität Göttingen

21.11.1998, Textgestaltung fürs World Wide Web, Die Zeitung im Spannungsfeld von Druck und Digitalisierung, Universität Trier

Prof. Dr. Bruno Strecker

19.1.1998, Die methodische und sachliche Ausrichtung der „Grammatik der deutschen Sprache", Universität Gießen

14.9.1998, Die "Grammatik der deutschen Sprache". Eine kurze Präsentation, Germanistentreffen Deutschland-Spanien-Portugal des DAAD, Leipzig

15.9.1998, Grammatik aus funktionaler Sicht im DaF-Unterricht, Germanistentreffen Deutschland-Spanien-Portugal des DAAD, Leipzig

Dr. Wolfgang Teubert

1.5.1998, Property, Work, Natural Law and Human Rights. A Case Study in Historical Semantics, Sydney Linguistics Circle, Sydney/Australien 8.5.1998 (1) New Directions in Corpus Linguistics, (2) Corpus Linguistics at the Institut für deutsche Sprache, (3) Ontologies, Concepts and Translation, Kolloquium „Corpus Linguistics“, Department of English and Linguistics, Macquaire University, Sydney/Australien

21.5.1998, Die Entpluralisierung des Diskurses. Zur Entwicklung der deutschen Sprachkultur nach der Wiedervereinigung, German Studies, Macquarie University, Sydney/Australien

19.06.1998, Translation and the Dictionary, Tuscan Word Center, Vellano/Italien 
25.8.1998, Tools for Terminology Mining. Integration of Translation Tools in Terminography, ProCom '98, Wien/Österreich

6.10.1998, Language Resources, TELRI, and Multilingual Lexical Semantics, Conference „Language Technologies for the Slovene Language", Ljubljana/Slovenien

27.10.1998, Multilingual Lexical Semantics and the Corpus, Projekt „Haltungen zu Europa - Attitudes Towards Europe“, University of Durham/Großbritannien

30.11.1998, Translation: Ontologies for Multilingual Applications? Jubiläumskongress Modelle der Übersetzung - Grundlagen für Methodik, Bewertung, Computermodellierung, Universität des Saarlandes, Saarbrücken

5.12.1998, Concepts, Ontologies and Translation, Österreichische Linguistentagung, Workshop „Constructive Linguistics“, Salzburg/Österreich

Dr. Annette Trabold

13.3.1998, Was nun? Wie kann man das Verhältnis Sprachwissenschaft - Öffentlichkeit verbessern? Podiumsdiskussion, 34. Jahrestagung des Instituts für deutsche Sprache, Mannheim

Klaus Vorderwülbecke

4.6.1998, Jedem das Seine - Medien und Landeskunde, Jahrestagung Deutsch als Fremdsprache, Jena

9.9.1998, Aktive und aktivierende Landeskunde, Universität Kutaissi/Georgien

10.9.1998, „Einblicke“ - ein neuer Fernsehsprachkurs, Universität Kutaissi/Georgien

Dr. Peter Wagener

31.3.1998, Digitale Archive in der empirischen Linguistik, Frühjahrstagung der Medienarchivare im Verband deutscher Archivare, Würzburg

2.6.1998, Niederdeutsch im Wandel - eine Panelstudie in realer Zeit, Jahrestagung des Vereins für niederdeutsche Sprachforschung, Göttingen

10.9.1998, Varianten des Deutschen, Seminar für Sprachmethodik, Burbach

17.11.1998, The Digital Sound Archives of Spoken German (PC-Präsentation), Annual Conference of the International Association of Sound Archives, Paris/Frankreich

Prof. Dr. Gisela Zifonun

15.5.1998, Theoretische Grundlagen der Valenz, Workshop „Valenzen, Argumente und Szenen“, IDS, Mannheim 


\section{Im Berichtsjahr erschienene Publikationen von IDS-Mitarbeitern}

Prof. Dr. Joachim Ballweg

Ballweg, Joachim (1998): Eine einheitliche Interpretation des attributiven Genitivs. In: Vuillaume, Marcel (Hg.): Der Kasus im Deutschen. Form und Inhalt. Tübingen. S. 153-168. (Eurogermanistik 13).

Ballweg, Joachim (1998): Zeit, Temporalität und Sprache. In: Hess-Lüttich, Ernest W./Schlieben-Lange, Brigitte (Hg.): Signs \& Time/Zeit \& Zeichen. An International Conference on the Semiotics of Time in Tübingen. Tübingen. S. 41-57.

Ballweg, Joachim (1997): Zusammengesetzte Tempora und dynamische Tempusinterpretation. In: Quintin, Herve/Najar, Margarete/Genz, Stephanie (Hg.): Temporale Bedeutungen - Temporale Relationen. Tübingen. S. 59-68. (Eurogermanistik 11).

Dr. Birgit Barden

Barden, Birgit/Großkopf, Beate (1998): Sprachliche Akkommodation und soziale Integration. Sächsische Übersiedler und Übersiedlerinnen im rhein-/moselfränkischen und alemannischen Sprachraum. Tübingen. $526 \mathrm{~S}$. (Phonai 43).

Auer, Peter/Barden, Birgit/Großkopf, Beate (1998): Subjective and objective parameters determining ,,salience" in long-term dialect accommodation. In: Journal of Sociolinguistics 2/2, S. 163-188.

Selting, Margret/Auer, Peter/Barden, Birgit/Bergmann, Jörg/CouperKuhlen, Elizabeth/Günthner, Susanne/Meier, Christoph/Quasthoff, Uta/Schlobinski, Peter/Uhmann, Susanne (1998): Gesprächsanalytisches Transkriptionssystem (GAT). In: Linguistische Berichte 173, S. 91-122.

Dr. Karl-Heinz Bausch

Bausch, Karl-Heinz (1998): „Sie hat Udo geküsst“ oder: Klatsch-Variationen. Verbale Strategien und Sprachvarianz unter Jugendlichen. In: Henn-Memmesheimer, Beate (Hg.): Sprachliche Varianz als Ergebnis von Handlungswahl. Tübingen. S. 135-155. (Reihe Germanistische Linguistik 198).

Cyril Belica

Belica, Cyril (1998): Statistische Analyse von Zeitstrukturen in Korpora. In: Teubert, Wolfgang (Hg.): Neologie und Korpus. Tübingen. S. 31-42. (Studien zur deutschen Sprache 11).

Dr. Ursula Brauße

Brauße, Ursula (1998): Was ist Adversativität? Aber oder und? In: Deutsche Sprache 26/2, S. 183-159. 
Dr. Eva Breindl

Breindl, Eva (1998): DaF goes Internet! Neue Entwicklungen in Deutsch als Fremdsprache. In: Deutsche Sprache 25/4, S. 288-342.

Breindl, Eva (1998): Konzeption und Konversion: zur simultanen Produktion von Printtext und Hypertext am Beispiel Grammatik. In: Storrer, Angelika/Harriehausen, Bettina (Hg.): Hypermedia für Lexikon und Grammatik. Tübingen. S. 75-98. (Studien zur deutschen Sprache 12).

Bahlmann, Clemens/Breindl, Eva/Dräxler, Hans-Dieter/Ende, Karin/ Storch, Günter (1998): Unterwegs. Lehrwerk für die Mittelstufe Deutsch als Fremdsprache. Berlin u. a. (darin: S. 129-164: Übersicht über Grammatik und Redemittel).

Dr. Elke Donalies

Donalies, Elke (1998): Der Basler-Nachlaß. In: Deutsche Sprache 25/4, S. 383-384.

Prof. Dr. Reinhard Fiehler

Fiehler, Reinhard (1998): Bewertungen und Normen als Problem bei der Förderung von Gesprächsfähigkeiten. In: Der Deutschunterricht 50/1, S. 53-64.

Fiehler, Reinhard (1998): Kommunikation im Alter. Drei Zugänge zur Analyse altersspezifischen Kommunikationsverhaltens. In: Reiher, Ruth/Kramer, Undine (Hg.): Sprache als Mittel von Identifikation und Distanzierung. Frankfurt/M. S. 299-317.

Fiehler, Reinhard (1998): Modelle zur Beschreibung und Erklärung altersspezifischer Sprache und Kommunikation. In: Fiehler, Reinhard/Thimm, Caja (Hg.): Sprache und Kommunikation im Alter. Opladen/Wiesbaden. S. 38-56.

Brünner, Gisela/Fiehler, Reinhard (1998): Linguistische Untersuchungen zur Wirtschaftskommunikation. In: Sprachreport 3, S. 13-16.

Fiehler, Reinhard/Thimm, Caja (1998): Das Alter als Gegenstand linguistischer Forschung - eine Einführung in die Thematik. In: Fiehler, Reinhard/Thimm, Caja (Hg.): Sprache und Kommunikation im Alter. Opladen/Wiesbaden. S. 7-16.

Fiehler, Reinhard/Thimm, Caja (Hg.) (1998): Sprache und Kommunikation im Alter. Opladen/Wiesbaden. $314 \mathrm{~S}$.

PD Dr. Claudia Fraas

Fraas, Claudia (1998): Interpretations- und Gebrauchsmuster abstrakter Nomina - ein korpusbasierter Beschreibungsansatz. In: Deutsche Sprache 26/3, S. 256-272.

Fraas, Claudia (1998): Karrieren geschichtlicher Grundbegriffe - FREIHEIT, GLEICHHEIT, BRÜDERLICHKEIT. In: Sprachreport 4, S. $2-10$. 
Fraas, Claudia (1998): Lexikalisch-semantische Eigenschaften von Fachsprachen. In: Hoffmann, Lothar/Kalverkämper, Hartwig/Wiegand, Herbert Ernst (Hg.): Fachsprachen - Languages for Special Purposes - Ein internationales Handbuch zur Fachsprachenforschung und Terminologiewissenschaft. Berlin/New York. S. 428-438. (Handbücher zur Sprach- und Kommunikationswissenschaft 14.1).

Haß-Zumkehr, Ulrike/Fraas, Claudia (1998): LEKSIS wissen über wörter. $\mathrm{Zu}$ einem neuen lexikografischen Vorhaben des Instituts für deutsche Sprache. http://www.ids-mannheim.de/leksis/

\section{Prof. Dr. Gisela Harras}

Harras, Gisela (1998): Intertextualität von linguistischen Fachtexten: ein Analysebeispiel. In: Hoffmann, Lothar/Kalverkämper, Hartwig/Wiegand, Herbert Ernst (Hg.): Fachsprachen - Languages for Special Purposes - Ein internationales Handbuch zur Fachsprachenforschung und Terminologiewissenschaft. Berlin/New York. S. 602-610. (Handbücher zur Sprach- und Kommunikationswissenschaft 14.1).

Harras, Gisela (1998): Jenseits von semantischen Konventionen - zum Beispiel: tautologische Äußerungen. In: Zeitschrift für germanistische Linguistik 26, S. 308-319.

Harras, Gisela/Grabowski, Joachim (1998): Zur Polysemie lokaler Präpositionen: Die Fragwürdigkeit von kategorialen Akzeptanzurteilen als Grundlage für bedeutungsbeschreibende Prototypenstrukturen. In: Manggasser-Wahl, Martina (Hg.): Prototypentheorie und Linguistik. Tübingen. S. 58-84.

Harras, Gisela/Großkopf, Anja (1998): Möglichkeiten der semantischen Erkundung von Sprechaktverben. In: Stumme, Gerd/Wille, Rudolf (Hg.): Begriffliche Wissensverarbeitung. Frankfurt/M. S. 127-151.

PD Dr. Ulrike Haß-Zumkehr

Haß-Zumkehr, Ulrike (1998): Einheit aus der Geschichte. Zur Erforschung der Geschichte der germanistischen Sprachwissenschaft. In: Mitteilungen. Marbacher Arbeitskreis für Geschichte der Germanistik 13/14, S. 11-23.

Haß-Zumkehr, Ulrike (1998): Die Fachsprache der Ökologie im 20. Jahrhundert. In: Hoffmann, Lothar/Kalverkämper, Hartwig/Wiegand, Herbert Ernst (Hg.): Fachsprachen - Languages for Special Purposes - Ein internationales Handbuch zur Fachsprachenforschung und Terminologiewissenschaft. Berlin/New York. S. 1363-1369. (Handbücher zur Sprach- und Kommunikationswissenschaft 14.1).

Haß-Zumkehr, Ulrike (1998): Für den Wechsel im Wandel. Zukunft als nachhaltige Verantwortungsphase. Eine Blütenlese der Parteiprogramme zur Bundestagswahl 1998. In: Sprachreport 3, S. 2-7. 
Haß-Zumkehr, Ulrike (1998): Die gesellschaftlichen Interessen an der Sprachgeschichte im 19. und 20. Jahrhundert. In: Besch, Werner/Betten, Anne/Reichmann, Oskar/Sonderegger, Stefan (Hg.): Sprachgeschichte. Ein Handbuch. 2. vollständig neu bearb. Aufl., 1. Teilbd. Berlin/New York. S. 349-358. (Handbücher zur Sprach- und Kommunikationswissenschaft 2.1).

Haß-Zumkehr, Ulrike (1998): Die Weimarer Reichsverfassung - Tradition, Funktion, Rezeption. In: Kämper, Heidrun/Schmidt, Hartmut (Hg.): Das 20. Jahrhundert. Sprachgeschichte - Zeitgeschichte. Jahrbuch 1997 des Instituts für deutsche Sprache. Berlin/New York. S. 225-249. (Jahrbücher des Instituts für deutsche Sprache).

Haß-Zumkehr, Ulrike (1998): ,Wie glaubwürdige Nachrichten versichert haben". Formulierungstraditionen in Zeitungsnachrichten des 17. bis 20. Jahrhunderts. Tübingen. $240 \mathrm{~S}$. (Studien zur deutschen Sprache 13). Haß-Zumkehr, Ulrike (1998): Zur sprachlichen Gestaltung von Mahnmalen und Gedenkstätten.In: Haase, Norbert/Pampel,Bert(Hg.): Doppelte Last - doppelte Herausforderung. Gedenkstättenarbeit und Diktaturenvergleich an Orten mit doppelter Vergangenheit. Frankfurt/M. S. 147-151.

Haß-Zumkehr, Ulrike/Fraas, Claudia (1998): LEKSIS wissen über wörter. $\mathrm{Zu}$ einem neuen lexikografischen Vorhaben des Instituts für deutsche Sprache. http://www.ids-mannheim.de/leksis/

\section{Dr. Klaus Heller}

Heller, Klaus (1998): Schifffahrt Starnberger See. Sprachglosse. In: Sprachreport 3, S. 25.

Heller, Klaus (1997/1998): Zum Stand der Orthographie-Reform. Deutschland. In: Mitteilungsheft 1997/98 der Internationalen Vereinigung für Germanische Sprach- und Literaturwissenschaft (IVG), S. 7-10.

Heller, Klaus (1998): Rechtschreibreform: Turbulenzen. Und wie weiter? In: Mitteilungen des Deutschen Germanistenverbandes 4, S. 122-124. Heller, Klaus (1998): Keine „Reform der Reform“. Die Vorschläge der zwischenstaatlichen Rechtschreibkommission. In: SchulVerwaltung Zeitschrift für SchulLeitung, SchulAufsicht und SchulKultur. Ausgabe Nordrhein-Westfalen, 3, März 1998, S. 67-68.

(Nachdrucke in:

SchulVerwaltung. Ausgabe Rheinland-Pfalz und Saarland 3, März 1998, S. 43-44,

,aktuelles“ (Oslo) 23, April 1998, S. 10-12,

FHTW-Informationsblatt der Fachhochschule für Technik und Wirtschaft Berlin, 2. Ausg., April 1998, S. 23.)

Heller, Klaus (1998): Mehr Freiheit für den Bindestrich. In: Lingua. Zeitschrift des schwedischen Fremdsprachenlehrerverbandes (LMS), 1, S. 43 (Nachdruck aus: DAAD Letter. Hochschule und Ausland 4/1997). 
(Nachdruck in:

FHTW-Informationsblatt der Fachhochschule für Technik und Wirtschaft Berlin, 3. Ausg., Juni 1998, S. 25).

Heller, Klaus (1998): Klein und groß für Groß und Klein. In: FHTWInformationsblatt der Fachhochschule für Technik und Wirtschaft Berlin, 1. Ausg., Januar 1998, S. 19 (= Nachdruck aus: DAAD Letter 3/97).

Heller, Klaus (1998): Rezension zu: Lívia Mádl-Pálfy und Beáta TujnerMarkó: Kaspertheater rund ums Jahr. Stuttgart 1996. In: Deutsch als Fremdsprache 2/2. Quartal 1998, S. 117-118.

Heller, Klaus (1998): Pro \& Kontra: Rechtschreibreform. Pro. In: Allgemeine LehrerZeitung (ALZ), Mai 1998, S. 2.

Heller, Klaus (1998): Bürgerkrieg ums Komma. In: Lingua. Zeitschrift des schwedischen Fremdsprachenlehrerverbandes (LMS) 3, S. 40-42.

Heller, Klaus (1998): „Erleichternd und zukunftsweisend“. In: Der Tagesspiegel, 31. Juli 1998, S. 7.

Heller, Klaus (1998): Die Neuerungen auf einen Blick. In: Verwaltung in Fahrt (ViF), Zeitschrift für die Mitarbeiterinnen und Mitarbeiter der Landesverwaltung Niedersachsen, 3, September/Oktober 1998, Sonderbeilage zur Rechtschreibreform, S. 2-4 (= Nachdruck der Sprachreport-Extraausgabe vom Juli 1996).

Heller, Klaus (1998): Rechtschreibreform. Eine Zusammenfassung von Dr. Klaus Heller. Sprachreport-Extraausgabe, Oktober 1998. Herausgegeben vom Institut fuir deutsche Sprache, Mannheim. (Aktualisierter Nachdruck der Ausgabe vom Juli 1996. Inhaltliche Fragen der Neuregelung sind nicht berührt.)

(Nachdrucke u. a.:

als Merkblatt der Senatsverwaltung für Inneres, Berlin,

als Kunstkalender „Rechtschreibreform" für die Jahre 1999 und 2000.

Verkaufsförderung Borm. Hildesheim 1998,

als Sonderdruck „Neue deutsche Rechtschreibung“ für die Verwaltung der

Stadt Duisburg,

als Teil eines Sonderdruckes des Enzyklopädischen Verlages Dr. phil.

Egon Müller GmbH, Salzburg „Die neue Rechtschreibung“, Salzburg 1998, S. 4-22,

als Broschüre „Die wichtigsten Regeln der neuen Rechtschreibung“,

Dussmann - das KulturKaufhaus, Berlin, als Informationsbroschüre der Deutschen Bundesbahn, als Sonderdruck des Bundesverwaltungsamtes).

Dr. Manfred Hellmann

Hellmann, Manfred W. (1998): Allgemeinwortschatz der DDR-Bürger.

Rezension. In: Sprachreport 2, S. 14-16. 
Prof. Dr. Dieter Herberg

Herberg, Dieter (1998): Auf dem Weg zum deutschen Neologismenwörterbuch. In: Zettersten, Arne/Pedersen, Viggo Hjørnager/Mogensen, Jens Erik (Hg.): Symposium on Lexicography VIII. S. 191-196. (Lexicographica Series Maior 90).

Herberg, Dieter (1998): DieAußentexte in Langenscheidts Großwörterbuch Deutsch als Fremdsprache. In: Wiegand, Herbert Ernst (Hg.): Perspektiven der pädagogischen Lexikographie des Deutschen. Untersuchungen anhand von „Langenscheidts Großwörterbuch Deutsch als Fremdsprache“. Tübingen. S. 331-341. (Lexicographica. Series Maior 86).

Herberg, Dieter (1998): Neues im Wortgebrauch der Wendezeit. Zur Arbeit mit dem IDS-Wendekorpus. In: Teubert, Wolfgang (Hg.): Neologie und Korpus. Tübingen. S. 43-62. (Studien zur deutschen Sprache 11).

Herberg, Dieter (1998): Schlüsselwörter - Schlüssel zur Wendezeit. In: Kämper, Heidrun/Schmidt, Hartmut (Hg.): Das 20. Jahrhundert. Sprachgeschichte - Zeitgeschichte. Jahrbuch 1997 des Instituts für deutsche Sprache. Berlin/New York. S. 330-344. (Jahrbücher des Instituts für deutsche Sprache).

Herberg, Dieter/Kinne, Michael (1998): Neologismen. Heidelberg. 48 S. (Studienbibliographien Sprachwissenschaft 23).

Claus Hoffmann

Hoffmann, Claus (1998): Das „Inferno“ im Wohnzimmer. Sprachglosse. In: Sprachreport 2, S. 17.

Gabriele Hoppe

Hoppe, Gabriele (1998): Herausbildung und Integration des Submusters ETHNIKA + -(o)phone/-(o)phonie im Französischen und Stellung des analogen Musters im Lehnwortbildungssystem des Deutschen. $90 \mathrm{~S}$. (amades - Arbeitspapiere und Materialien zur deutschen Sprache 0).

Dr. Heidrun Kämper

Kämper, Heidrun (1998): Entnazifizierung - Sprachliche Existenzformen eines ethischen Konzepts. In: Kämper, Heidrun/Schmidt, Hartmut (Hg.): Das 20. Jahrhundert. Sprachgeschichte - Zeitgeschichte. Jahrbuch 1997 des Instituts für deutsche Sprache. Berlin/New York. S. 304-329. (Jahrbücher des Instituts für deutsche Sprache).

Kämper, Heidrun (1998): Das Korpus des Deutschen Fremdwörterbuchs. In: Bergmann, Rolf (Hg.) im Auftrag der Kommission für das deutsche Wörterbuch von Jacob und Wilhelm Grimm: Probleme der Textauswahl für einen elektronischen Thesaurus. Beiträge zum ersten Göttinger Arbeitsgespräch zur historischen deutschen Wortforschung, 1. und 2. November 1996. S. 57-68.

Kämper, Heidrun (1998): Politische Leitvokabeln. (Rezension von: Karin 
Böke/Frank Liedtke/Martin Wengeler: Politische Leitvokabeln in der Adenauer-Ära. Mit einem Beitrag von Dorothee Dengel. Berlin/New York 1996). In: Sprachreport 3, S. 16-19.

Kämper, Heidrun/Schmidt, Hartmut (1998): Vorwort. In: Kämper, Heidrun/Schmidt, Hartmut (Hg.): Das 20. Jahrhundert. Sprachgeschichte Zeitgeschichte. Jahrbuch 1997 des Instituts für deutsche Sprache. Berlin/New York. S. 1-4. (Jahrbücher des Instituts für deutsche Sprache).

Kämper, Heidrun/Schmidt, Hartmut (Hg.) (1998): Das 20. Jahrhundert. Sprachgeschichte - Zeitgeschichte. Jahrbuch 1997 des Instituts für deutsche Sprache. Berlin/New York. 446 S. (Jahrbücher des Instituts für deutsche Sprache).

PD Dr. Inken Keim

Keim, Inken (1998): Herstellen von Dominanz im Gespräch durch Dominantsetzen von Perspektiven. In: Fohrmann, Jürgen/Kasten, Ingrid/Neuland, Eva (Hg.): Autorität und Sprache. Im Druck.

Keim, Inken (1998): Werbeverhalten und sozialer Stil. In: Schoenthal, Gisela (Hg.): Feministische Linguistik - Linguistische Geschlechterforschung. S. 221-252. (Germanistische Linguistik 139-140).

Dr. Michael Kinne

Kinne, Michael (1998): Der lange Weg zum deutschen Neologismenwörterbuch. Neologismus und Neologismenlexikographie im Deutschen: Zur Forschungsgeschichte und zur Terminologie, über Vorbilder und Aufgaben. In: Teubert, Wolfgang (Hg.): Neologie und Korpus. Tübingen. S. 63-110. (Studien zur deutschen Sprache 11).

Herberg, Dieter/Kinne, Michael (1998): Neologismen. Heidelberg. 48 S. (Studienbibliographien Sprachwissenschaft 23).

Jacqueline Kubczak

Kubczak, Jacqueline (1998): Valenz in zweisprachigen Wörterbüchern Deutsch-Französisch/Französisch-Deutsch. In: Bresson, Daniel (Hg.): Lexikologie und Lexikographie Deutsch-Französisch. Aix-en-Provence. S. 65-78. (Cahiers d'Etudes germaniques 35).

Kubczak, Jacqueline (1998): Vorwort der Herausgeber. In: Bresson, Daniel/Kubczak, Jacqueline (Hg.): Abstrakte Nomina. Vorarbeiten zu ihrer Erfassung in einem zweisprachigen syntagmatischen Wörterbuch. Tübingen. S. 7-10. (Studien zur deutschen Sprache 10).

Bresson, Daniel/Kubczak, Jacqueline (Hg.) (1998): Abstrakte Nomina. Vorarbeiten zu ihrer Erfassung in einem zweisprachigen syntagmatischen Wörterbuch. Tübingen. 300 S. (Studien zur deutschen Sprache 10).

Kubczak, Jacqueline/Costantino, Sylvie (1998): Exemplarische Untersuchungen für ein syntagmatisches Wörterbuch Deutsch-Französisch/ Französisch-Deutsch. In: Bresson, Daniel/Kubczak, Jacqueline (Hg.): 
Abstrakte Nomina. Vorarbeiten zu ihrer Erfassung in einem zweisprachigen syntagmatischen Wörterbuch. Tübingen. S. 11-120. (Studien zur deutschen Sprache 10).

Kubczak, Jacqueline/Costantino, Sylvie (1998): Frage (Frage / Frage- / -frage). Über ein französisch-deutsches Kooperationsprojekt: Syntagmatisches Wörterbuch (Lexique-Grammaire) Deutsch-Französisch, Französisch-Deutsch. In: Bassola, Peter ( $\mathrm{Hg}$.): Beiträge zur Nominalphrasensyntax. Szeged. S. 63-128. (Acta Germanica 6).

Kubczak, Jacqueline/Schumacher, Helmut (1998): Verbvalenz - Nominalvalenz. In: Bresson, Daniel/Kubczak, Jacqueline (Hg.): Abstrakte Nomina. Vorarbeiten zu ihrer Erfassung in einem zweisprachigen syntagmatischen Wörterbuch. Tübingen. S. 273-286. (Studien zur deutschen Sprache 10).

Dr. Ann Lawson

Lawson, Ann (1998): The Bridge Dictionary Multilingual Database. http://solaris3.ids-mannheim.de/workshop/lawson.html

Lawson, Ann (1998): English Words in Use: Compiling a Dictionary of Collocation. In: PALC '97. Practical Applications of Language Corpora. Lodz. S. 225-235.

Erjavec, Tomaz/Lawson, Ann/Romary, Laurent (Hg.) (1998): East meets West - A Compendium of Multilingual Resources. CD-ROM.

Pantelis Nikitopoulos

Nikitopoulos, Pantelis (1998): Griechisch als Fremdsprache in der deutschen Gesellschaft. Studie über mögliche Benutzer der griechischen Sprache (Gebiete - Institutionen) und Strategien zur Förderung von Griechisch als Fremdsprache. (Untersuchung im Rahmen des EU-Projekts „Griechischsprachige Erziehung in Deutschland“ - Sokrates-Programm), Pädagogisches Institut Mittelhessen/Fachberatungszentrum Frankfurt/M. (griech.).

Nikitopoulos, Pantelis et al. (1998): Sprechen statt schlagen. Erwachsenenbildung im Strafvollzug. Erhöhung der Resozialisierungschancen durch Erweiterung der sprachlichen und kommunikativen Kompetenz. Ein methodischer Leitfaden. (Transnationales Pilotprojekt im Rahmen des Sokrates-Förderprogramms der EU). Mannheim/Blackpool/Palermo.

Wolfgang Mentrup

Mentrup, Wolfgang (1998): Neuregelung der Rechtschreibung. Zur Anhörung des Bundesverfassungsgerichtes (Karlsruhe 12. Mai 1998) Impressionen - Argumentationen. $10 \mathrm{~S}$. http://www.ids-mannheim.de/reform/bvg5-98.doc.

Dr. Renate Pasch

Kempcke, Günter/Pasch, Renate (1998): Die Konjunktionen in Langen- 
scheidts Großwörterbuch Deutsch als Fremdsprache. In: Wiegand, Herbert Ernst (Hg.): Perspektiven der pädagogischen Lexikographie des Deutschen. Untersuchungen anhand von „Langenscheidts Großwörterbuch Deutsch als Fremdsprache“. Tübingen. S. 233-243. (Lexicographica. Series Maior 86).

Prof. Dr. Joachim Schildt

Schildt, Joachim (1998): Deutsche Sprachgeschichte und Geschichte von Institutionen. In: Besch, Werner/Betten, Anne/Reichmann, Oskar/Sonderegger, Stefan (Hg.): Sprachgeschichte. Ein Handbuch zur Geschichte der deutschen Sprache und ihrer Erforschung. Bd. 1, 2. Aufl. Berlin' New York. S. 55-62. (Handbücher zur Sprach- und Kommunikationswissenschaft 2.1).

Schildt, Joachim (1998): Frühneuhochdeutsches Wörterbuch. Hg. von Ulrich Goebel/Oskar Reichmann in Verbindung mit dem Institut für deutsche Sprache. Berlin/New York. Bd. 4, Lfg. 2 (plagen-brechen).

Schildt, Joachim (1998): Modalwörter in Luther-Schriften. In: Kommunikationspraxis und ihre Reflexion in frühneuhochdeutscher und neuhochdeutscher Zeit. Festschrift für Monika Rössing-Hager zum 65. Geburtstag. S. 67-73. (Germanistische Linguistik. Monographien 2).

Prof. Dr. Hartmut Schmidt

Schmidt, Hartmut (1998): „An mein Volk“. Sprachliche Mittel monarchischer Appelle. In: Cherubim, Dieter/Grosse, Siegfried/Mattheier, Klaus J. (Hg.): Sprache und bürgerliche Nation. Beiträge zur deutschen und europäischen Sprachgeschichte des 19. Jahrhunderts. Berlin/New York. S. 167-196.

Schmidt, Hartmut (1998): Rezension über: G. Fritz \& E. Straßner (1996): Die Sprache der ersten deutschen Wochenzeitungen im 17. Jahrhundert. Tübingen. In: Leuvense Bijdragen 87, S. 248-250.

Schmidt, Hartmut (1998): Rezension über: U. Goebel, I. Lemberg \& O. Reichmann (1995): Versteckte lexikographische Information. Möglichkeiten ihrer Erschließung dargestellt am Beispiel des Frühneuhochdeutschen Wörterbuchs. Tübingen. In: Leuvense Bijdragen 87, S. 245-247.

Schmidt, Hartmut (1998): Traditionen des Formulierens: Apposition, Triade, Alliteration, Variation. In: Kämper, Heidrun/Schmidt, Hartmut (Hg.): Das 20. Jahrhundert. Sprachgeschichte - Zeitgeschichte. Jahrbuch 1997 des Instituts für deutsche Sprache. Berlin/New York. S. 86-117. (Jahrbücher des Instituts für deutsche Sprache).

Kämper, Heidrun/Schmidt, Hartmut (1998): Vorwort. In: Kämper, Heidrun/Schmidt, Hartmut (Hg.): Das 20. Jahrhundert. Sprachgeschichte - Zeitgeschichte. Jahrbuch 1997 des Instituts für deutsche Sprache. Berlin/New York. S. 1-4. (Jahrbücher des Instituts für deutsche Sprache). 
Kämper, Heidrun/Schmidt, Hartmut (Hg.) (1998): Das 20. Jahrhundert. Sprachgeschichte - Zeitgeschichte. Jahrbuch 1997 des Instituts für deutsche Sprache. Berlin/New York. 446 S. (Jahrbücher des Instituts für deutsche Sprache).

Dr. Reinhold Schmitt

Schmitt, Reinhold (1998): The Making of „Körperwelten“: Arbeit und Kommunikation im Hintergrund einer Ausstellung. In: Sprachreport 3, S. 8-13.

Schmitt, Reinhold (1998): Unterstützen im Gespräch. Zur Analyse manifester Kooperationsverfahren. In: Zeitschrift für Sprachwissenschaft $16 / 1-2$, S. 52-82.

Liebert, Wolf-Andreas/Schmitt, Reinhold (1998): Texten als Dienstleistung. Sprachwissenschaftler schreiben die besseren Gebrauchsanweisungen. In: Sprachreport 1, S. 1-5.

\section{Dr. Rosemarie Schnerrer}

Schnerrer, Rosemarie (1998): ,jetzt schlägt's dreizehn“. Woher kommt dieser Ausdruck und was bedeutet er? In: Sprachreport 2, S. 6.

Schnerrer, Rosemarie (1998): Nachgefragt: "Globalisierung“. Woher kommt der Ausdruck und was bedeutet er? In: Sprachreport 3, S. 23-25.

\section{Helmut Schumacher}

Schumacher, Helmut (1998): Über den Nutzen von Grundwortschätzen. In: Jung, Udo O. H. (Hg.): Praktische Handreichung für Fremdsprachenlehrer. 2., verbesserte und erweiterte Auflage unter Mitarbeit von Heidrun Jung. Frankfurt/M. S. 161-168. (Bayreuther Beiträge zur Glottodidaktik 2).

Schumacher, Helmut (1998): Valenz - peijia. Zur Valenzforschung im Deutschen und Chinesischen. Dokumentation. In: Deutsche Sprache 26/2, S. 190.

Kubczak, Jacqueline/Schumacher, Helmut (1998): Verbvalenz - Nominalvalenz. In: Bresson, Daniel/Kubczak, Jacqueline (Hg.): Abstrakte Nomina. Vorarbeiten zu ihrer Erfassung in einem zweisprachigen syntagmatischen Wörterbuch. Tübingen. S. 273-286. (Studien zur deutschen Sprache 10).

\section{PD Dr. Thomas Spranz-Fogasy}

Spranz-Fogasy, Thomas/Deppermann, Arnulf (1998): Kommunikationsstörungen durch den Gesprächsprozeß. Zur Entstehung von Interaktionsdilemmata durch zeitliche Komplexierung. In: Fiehler, Reinhard (Hg.): Verständigungsprobleme und gestörte Kommunikation. Opladen. S. 44-62. 
Dr. Kathrin Steyer

Steyer, Kathrin (1998): Kollokationen als zentrales Übersetzungsproblem - Vorschläge für eine Kollokationsdatenbank Deutsch-Französisch/ Französisch-Deutsch auf der Basis paralleler und vergleichbarer Korpora. In: Bresson, Daniel ( $\mathrm{Hg}$.): Lexikologie und Lexikographie Deutsch-Französisch. Aix-en-Provence. S. 95-113. (Cahiers d'Études Germaniques 35).

Steyer, Kathrin (1998): Sprachstrategien und Dialogblockaden. In: Sprachreport 1, S. 15-16.

Steyer, Kathrin/Teubert, Wolfgang (1998): Deutsch-Französische Übersetzungsplattform. Ansätze, Methoden, empirische Möglichkeiten. In: Deutsche Sprache 25/4, S. 343-359.

Prof. Dr. Gerhard Stickel

Stickel, Gerhard (1998): Eröffnung der Jahrestagung 1997. In: Kämper, Heidrun/Schmidt, Hartmut (Hg.): Das 20. Jahrhundert. Sprachgeschichte - Zeitgeschichte. Jahrbuch 1997 des Instituts für deutsche Sprache. Berlin/New York. S. 5-8. (Jahrbücher des Instituts für deutsche Sprache). Stickel, Gerhard (1998): Der Sprachfeminismus geht in die falsche Richtung. In: Brunner, Margot/Frank-Cyrus, Karin M. (Hg.): Die Frau in der Sprache. Wiesbaden. S. 64-76.

Stickel, Gerhard (1998): Zum Streit um die Reform der deutschen Rechtschreibung. In: Leksika i leksikografika 9. (Rossija Akademija Nauk, Institut Jazikoznanija). S. 150-167.

Dr. Angelika Storrer

Storrer, Angelika (1998): Hypermedia als Kommunikations- und Publikationsmedium in den Geisteswissenschaften. In: Sprache und Datenverarbeitung, 2/1, S. 5-9.

Storrer, Angelika (1998): Hypermedia und Grammatikographie. In: Storrer, Angelika/Harriehausen, Bettina (Hg.): Hypermedia für Lexikon und Grammatik. Tübingen. S. 29-56. (Studien zur deutschen Sprache 12).

Storrer, Angelika (1998): Hypermedia-Wörterbücher: Perspektiven für eine neue Generation elektronischer Wörterbücher. In: Wiegand, Herbert Ernst $(\mathrm{Hg}$.): Wörterbücher in der Diskussion III. Tübingen. S. 106-131. (Lexicographica Series Maior).

Harriehausen, Bettina/Storrer, Angelika (1998): Einführung. In: Storrer, Angelika/Harriehausen, Bettina (Hg.): Hypermedia für Lexikon und Grammatik. Tübingen. S. 9-19. (Studien zur deutschen Sprache 12). Storrer, Angelika/Harriehausen, Bettina (Hg.) (1998): Hypermedia für Lexikon und Grammatik. 275 S. (Studien zur deutschen Sprache 12).

Storrer, Angelika/Schwall, Ulrike (1998): Computersystem und Verfahren zur automatischen Analyse eines Textes. Patentschrift DE $4311211 \mathrm{C} 2$. Deutsches Patentamt. Patentinhaber: IBM. 
Storrer, Angelika/Waldenberger, Sandra (1998): Zwischen Grice und Knigge: Die Netiketten im Internet. In: Hielscher, Martina/Sichelschmidt, Lorenz/Strohner, Hans (Hg.): Medium Sprache. Frankfurt/M. S. 63-77. http://www.ids-mannheim.de/grammis/netiketten/

Prof. Dr. Bruno Strecker

Strecker, Bruno (1998): Hypertext: Chance und Herausforderung für die Grammatikschreibung. In: Storrer, Angelika/Harriehausen, Bettina (Hg.): Hypermedia für Lexikon und Grammatik. S. 21-28. (Studien zur deutschen Sprache 12).

Strecker, Bruno (1998): Sprache in der Wissenschaft. In: WGL-Journal 4, S. $8-12$.

Dr. Wolfgang Teubert

Teubert, Wolfgang (1998): Eigentum, Arbeit, Naturrecht. Schlüsselwörter der Soziallehre im Wandel. In: Kämper, Heidrun/Schmidt, Hartmut (Hg.): Das 20. Jahrhundert. Sprachgeschichte - Zeitgeschichte. Jahrbuch 1997 des Instituts für deutsche Sprache. Berlin/New York. S. 188-224. (Jahrbücher des Instituts für deutsche Sprache).

Teubert, Wolfgang (1998): Europäische Herausforderungen. In: Sprachreport 4, S. 11-13.

Teubert, Wolfgang (1998): Korpus und Neologie. In: Teubert, Wolfgang (Hg.): Neologie und Korpus. Tübingen. S. 129-170. (Studien zur deutschen Sprache 11).

Teubert, Wolfgang (1998): Korpus und Terminologiearbeit. DIN NAT 5 Nr. 21-98. S. 1-3.

Teubert, Wolfgang (1998): Language Resources, TELRI, and Multilingual Lexical Semantics. In: Erjavec, Tomaz/Gros, Jerneja (Hg.): Jezikovne Teknologije Slovenski Jezik/Language Technologies for the Slovene Language. Proceedings. Ljubljana. S. 1-8.

Teubert, Wolfgang (Hg.) (1998): Neologie und Korpus. Tübingen. $170 \mathrm{~S}$. (Studien zur deutschen Sprache 11).

Teubert, Wolfgang (1998): Translation and the Dictionary. In: Papers of the Workshop Multilingual Lexical Semantics. Vellano. http://solaris3.ids-mannheim.de/workshop/teubert.html

Teubert, Wolfgang (1998): Übersetzung und Korpus. In: Cahiers d'Etudes germaniques 35 , S. 127-141.

Teubert, Wolfgang (1998): Vorwort. In: Teubert, Wolfgang (Hg.): Neologie und Korpus. Tübingen. S. 7-9. (Studien zur deutschen Sprache 11).

Teubert, Wolfgang (1998): Zum Verlust von Pluralität im politisch-gesellschaftlichen Diskurs: Das Beispiel Besitzstände. In: Kreft, Ursula u. a. (Hg.): Kassensturz. Politische Hypotheken der Berliner Republik. Duisburg. S. $29-48$.

Teubert, Wolfgang/Bonelli, Elena Tognini/Volz, Norbert (Hg.) (1998): 
„Translation Equivalence“. TELRI. Proceedings of the Third European

Seminar. Montecatini Terme, Italy (October 16-18, 1997). 276 S.

Teubert, Wolfgang/Kirsten Plöger (Hg.) (1998): TELRI (Trans-European Language Resources Infrastucture) Final Report. Mannheim/Birmingham [mit CD-ROM].

Steyer, Kathrin/Teubert, Wolfgang (1998): Deutsch-Französische Übersetzungsplattform. Ansätze, Methoden, empirische Möglichkeiten. In: Deutsche Sprache 25/4, S. 343-359.

Klaus Vorderwülbecke

Vorderwülbecke, Klaus (1998): Einblicke in „Einblicke“ - ein Fernsehsprachkurs mit einer Multimedia-Komponente. In: Storrer, Angeli$\mathrm{ka} /$ Harriehausen, Bettina (Hg.): Hypermedia für Lexikon und Grammatik. Tübingen. S. 131-144. (Studien zur deutschen Sprache 12).

Dr. Peter Wagener

Wagener, Peter (1998): Dialektologische Fachtagung Bredevoort und Doetinchem (14.-17.9.1996). In: Zeitschrift für Dialektologie und Linguistik LXV/1, S. 56-61.

Wagener, Peter (1998): Das Deutsche Spracharchiv. Forschungs- und Dokumentationszentrum für gesprochenes Deutsch. In: Verzeichnis der Dialektinstitute in der Bundesrepublik Deutschland. $\mathrm{Hg}$. vom Internationalen Mundartarchiv L. Soumagne. Neuß. S. 9-11.

Dr. Ricarda Wolf

Wolf, Ricarda (1998): Wo findet das Interkulturelle statt? Konversationsanalytische Überlegungen am Beispiel einer polnisch-deutschen Titelsuche. In: Apfelbaum, Birgit/Müller, Herrmann (Hg.): Fremde im Gespräch. Gesprächsanalytische Untersuchungen zu Dolmetschinteraktion, interkultureller Kommunikation und institutionalisierten Interaktionsformen. Frankfurt a. M. S. 111-143.

Prof. Dr. Gisela Zifonun

Zifonun, Gisela (1998): Zur Grammatik von Subsumtion und Identität. Herr Schulze als erfahrener Lehrer. In: Deutsche Sprache 26/1, S. 1-17.

\section{Kontakte des IDS zu anderen Institutionen, Studienaufenthalte und Besuche in- und ausländischer Wissenschaftler am IDS, Praktika, Besuchergruppen}

\subsection{Kontakte zu anderen Institutionen}

Alexander-von-Humboldt-Stiftung, Bonn

Arbeitskreis der Sprachzentren, Sprachlehrinstitute und Fremdspracheninstitute

Arbeitskreis für siebenbürgische Landeskunde, Gundelsheim 
Arbeitsstelle Deutsches Wörterbuch, Berlin

Arbeitsstelle Deutsches Wörterbuch, Göttingen

Arbeitsstelle „Sprache in Südwestdeutschland“, Tübingen

Berlin-Brandenburgische Akademie der Wissenschaften, Berlin

DANTE. Deutschsprachige Anwendervereinigung TEX e. V., Heidelberg

Deutsche Forschungsgemeinschaft (DFG), Bonn

Deutsche Gesellschaft für Sprachwissenschaft (DGfS)

Deutsche Gesellschaft für Dokumentation (DGD), Frankfurt a. M.

Deutscher Akademischer Austauschdienst (DAAD), Bonn

Deutscher Germanistenverband

Deutscher Sprachatlas, Marburg

DIN-Normenausschuß Terminologie, Berlin

Dudenredaktion des Bibliographischen Instituts, Mannheim

EURALEX, European Association for Lexicography, Exeter

Fachverband Deutsch als Fremdsprache

Fachverband Moderne Fremdsprachen

Forschungszentrum für die Landessprachen Finnlands, Helsinki

Fremdsprachenhochschule Tianjin, VR China

Fritz-Thyssen-Stiftung, Köln

GLDV, Gesellschaft für linguistische Datenverarbeitung, Frankfurt

Geisteswissenschaftliche Zentren, Berlin

Gesellschaft für angewandte Linguistik e. V. (GAL)

Gesellschaft für deutsche Sprache (GfdS), Wiesbaden

Gesellschaft für Mathematik und Datenverarbeitung mbH, Bonn

Goethe-Institut, München

Hugo-Moser-Stiftung im Stifterverband für die Deutsche Wissenschaft, Essen

Institut für Auslandsbeziehungen, Stuttgart

Inter Nationes, Bonn

International Association of Sound Archives (IASA)

Institut für niederdeutsche Sprache (INS), Bremen

Laboratoire d'Automatique Documentaire et Linguistique (LADL), Paris

Max-Planck-Institut für Psycholinguistik, Nijmegen

Österreichische Akademie der Wissenschaften, Wien

Polnische Akademie der Wissenschaften, Warschau

Robert-Bosch-Stiftung, Stuttgart

Russische Akademie der Wissenschaften, Institut für russische Sprache, Moskau

Universitäten Mannheim und Heidelberg sowie zahlreiche germanistische

Institute an weiteren Universitäten und Hochschulen im In- und Ausland Verein zur Förderung sprachwissenschaftlicher Studien, Berlin

Volkswagen-Stiftung, Hannover

Wissenschaftsgemeinschaft Gottfried Wilhelm Leibniz (WGL) 


\subsection{Studienaufenthalte und Besuche in- und ausländischer} Wissenschaftler am IDS

Anne Arold, Universität Tartu, Lehrstuhl für deutsche Philologie, Tartu, Estland, 1.4.-31.5.1998

Dr. habil. Peter Bassola, Joszef-Attila-Universität, Szeged, Ungarn, 27.4.30.5.1998 und 20.7.-20.8.1998

Federica Benacchio, Universität Udine, Udine, Italien, 23.10.-3.11.1998

Prof. Dr. Indu Bhave, Dept. of German Studies, Banaras Hindu Universität, Varanasi-5, Indien, 1.6.-6.8.1998

Dr. M. Teresa Bianco, Istituto Universitario Orientale, Napoli, Italien, 3. -28.8 .198$

Johanna Bottesch, Lucian-Blaga-Universität, Sibiu, Rumänien, 15.28.6.1998

Dr. Jun-Ku Cho, Sung Kyun Kwan University, German Department, Seoul, Korea, März 1998-März 1999

Dr. Leslaw Cirko, Universität Breslau, Institut für Germanistik, Breslau, Polen, 20.-27.7.1998

Ana Cletiu, Universität „1 Decembrie 1918“ Alba Iulia, Rumänien, 1.-14.7.1998

Philippa Cook, University of Manchester, German Dept., Manchester, England, 18.11.- 4.12.1998

Prof. Dr. Winifred Davies, University of Wales, Aberystwyth, Wales, England, 1.-22.4.1998

Dr. Boris Djubo, Russische Akademie der Wissenschaften, St. Petersburg, Rußland, 1.8.-31.10.1998

Yutaka Eguchi, Hokkaido Universität, Kita-Ku, Sapporo, Japan, 2.7.-1.10.1998

Prof. Dr. Sinaida Fomina, Universität Woronesh, Lehrstuhl für Deutsche Philologie, Woronesh, Rußland, 21.9.-21.11.1998

Dr. Joanna Golonka, Pädagogische Hochschule Rzeszow, Polen, 1.31.1.1998

Dr. Emo Gotsbachner, Universität Wien, Institut für Politikwissenschaften, Wien, Österreich, 22.-26.6.1998

Piklu Gautam Gupta, University of Manchester, England, 5.-24.8.1998

Gulnara Ischtuganowa, Baschkirische Staatliche Universität Ufa, Rußland, 1.10.1998-1.8.1999

Uta Itakura, M. A., Keio-Universität, Tokio, Japan, März 1998-März 1999 Makoto Itoh, University of Tsukuba, Institut für moderne Sprachen und Kulturen, Ibaraki, Japan, 1.4.1997-31.3.1998

Prof. Dr. Marja Järventausta, Universität Joensuu, Institut für interkulturelle Kommunikation, Savonlinna, Finnland, 5.-9.3.1998

Jörg Johannessohn, Mannheim, 4.5.--31.8.1998 
Dr. Sally Johnson, Lancester University, Dept. of Linguistics, Bowland College, Lancaster, England, 24.4.-24.7.1998

Dr. Ljubina Jordanova, Institut für bulgarische Sprache der Bulgarischen Akademie der Wissenschaften, Sofia, Bulgarien, 9.-7.6.1998

Milla Kajanne, M. A., Wirtschaftsuniversität Helsinki, Institut für Sprachen, Helsinki, Finnland, 7.1.-4.1998

Valerie Kervio-Berthou, Universität Nantes, Nantes, Frankreich, 1.2.31.3.1998

Dr. Olga Koleckova, Institut für Translatologie, Prag, Tschechien, 2.-16.10.1998

Uwe Kreisel, M.A., Universität Mannheim, 27.1.-31.12.1998

Prof. Dr. Sigmund Kvam, Gesamthochschule Østfold, Halden, Norwegen, 16.-3.6.1998

Dr. phil. Elzbieta Kucharska, M. A. Uniwersytet Wroclawski, Instytut Filologii Germanskiej, Wroclaw, Polen, 23.2.--2.3.1998

Cato Lambine, Germanistisches Institut, Universität Oslo, Norwegen, 2.-6.2.1998

Univ. Doz. Dongliang Li, Foreign Languages and Literature Dept., Hubei University, Wuhan, China, 9.6.-9.7.1998

Ingo Liebe, Institut für Germanistik, Universität Leipzig, 15.-17.6.1998

Meliha Mesanovic, Universität Tuzla, Jugoslawien, 28.1.--5.2.1998

Anna Molnar, Institut für Germanistik der Lajos-Kossuth-Universität, Debrecen, Ungarn, 5.10-11.1998

Prof. Dr. Pirkko Nuolijärvi, Forschungszentrum für die Landessprachen Finnlands, Helsinki, Finnland, 2.-7.11.1998

Aicha Ouaret, Universität Algier, Algier, Algerien, 27.6.-27.8.1998

Eliza Pieciul, Lehrstuhl für Glottodidaktik, Poznan, Polen, 5.5.-5.7.1998

Jewgenij Plissow, Linguistische Hochschule Nishnij Novgorod, Nishnij Novgorod, Russland, 1.9.-31.10.1998

Prof. Dr. Ekaterina Protassova, Pädagogische Universität Moskau, Moskau, Russland, 12.1.-12.3.1998

Lucile Salenson, Universität Aix-en-Provence, Institut für Germanistik, Aix-en-Provence, Frankreich, 16.-26.11.1998

Maria Smirnova, Moskauer Staatliche Lomonossow Universität, Moskau, Russland, 12.10.-31.12.1998

Prof. Dr. Pawan Surana, Universität Rajasthan, Jaipur, Indien, 12.5.15.7.1998

Prof. Dr. Speranta Stanescu, Universität Bukarest, Fakultät für Fremdsprachen, Bukarest, Rumänien, 1.5.-1.10.1998

Matthew Stevens, University of Auckland, Auckland, Neuseeland, 7.4.7.9.1998

Prof. Dr. Jürgen Streeck, College of Communication, University of Texas, Austin, USA, 1.-12.6.1998 
Prof. Dr. Liisa Tiittula, Universität Tampere, Tampere, Finnland, 2.30.11.1998

Prof. Dr. Michael Townson, School of Applied Language and Intercultural Studies, Dublin, Irland, 20.7.-5.8.1998

Prof. Dr. Yutaka Wakisaka, Tenri-Universität, Tenri-Shi, Japan, 19.8.31.10.1998

Lerleen Willis, The Nottingham Crest University, Nottingham, England, 10.-18.8.1998

Prof. Dr. Ruth Wodak, Institut für allgemeine und angewandte Linguistik, Universität Wien, Wien, Österreich, 14.-20.6.1998

Doz. Laila Zamzam, Universität Ain Sham Kairo, Kairo, Ägypten, 24.-31.7.1998

Dr. Erminka Zilic, Philosophische Fakultät der Universität Sarajevo, Bosnien, 16.4.-7.51998

\subsection{Praktika am IDS}

Ungeachtet der Tatsache, dass Praktika am IDS nicht vergütet werden können, ist ein wachsendes Interesse bei Studierenden an Praktika zu verzeichnen. In der Regel dauert ein Praktikum vier Wochen, die Studierenden sollten die Zwischenprüfung in Sprachwissenschaft abgelegt haben. Die Tätigkeitsbereiche werden nach Interesse der Studierenden von der Arbeitsstelle Öffentlichkeitsarbeit zusammengestellt.

1998 waren als Praktikanten am IDS:

Eva Susanne Breßler, 28.9.-16.10.1998; Frank Doerfeldt, 4.-29.5.1998; Markus Dreyer, 25.8.-14.9.1998; Natalia Fedossenko, 10.8.-4.9.1998; Brigitte Hammel, 20.10.-16.11.1998; Monika Hubele, 4.11.-1.12.1998; Carmen Kämmerer, 14.-25.9.1998; Birgit Kirchhoff, 14.5.-10.6.1998; Elisabeth Müller, 9.11.-19.3.1998; Evelin Naudorf, 27.7.-28.8.1998; Katrin Schmidt, 1.-30.9.1998; Gerd Steinmüller, 25.11.1997-1.4.1998

\subsection{Besuchergruppen}

Im Jahr 1998 waren 12 Besuchergruppen - zusammen rund 230 Personen - Gäste im IDS (die Gastwissenschaftlerinnen und Gastwissenschaftler nicht mitgezählt). Darunter waren der betriebswirtschaftliche Ausschuss der Industrie- und Handelskammer Rhein-Neckar, eine französische Studierendengruppe in Zusammenarbeit mit dem DAAD und dem Akademischen Auslandsamt der Universität Mannheim sowie Schülerinnen und Schüler einer gymnasialen Linguistik-Arbeitsgemeinschaft. Darüber hinaus informierten sich in- und ausländische Germanistik-Studierende von deutschen Universitäten im Rahmen ihrer Seminare über die Arbeit des IDS. Die Besuchergruppen erwartet ein möglichst auf ihre Interessen und Forschungsschwerpunkte zugeschnittenes Programm, das von einer allge- 
meinen Einführung in die Arbeit des IDS und von einer Bibliotheksführung eingerahmt wird.

\section{Gremien des Instituts für deutsche Sprache}

7.1 Wissenschaftlicher Beirat

Vorsitzender: Prof. Dr. Heinrich Löffler, Universität Basel

Stellvertreter: Prof. Dr. Ludwig Eichinger, Universität Kiel

Prof. Dr. Konrad Ehlich, Universität München - Prof. Dr. Peter Eisenberg, Universität Potsdam - Prof. Dr. Hans-Werner Eroms, Universität Passau - Prof. Dr. Cathrine Fabricius-Hansen, Universität Oslo - Prof. Dr. Hans Uszkoreit, Universität des Saarlandes - Prof. Dr. Peter Wiesinger, Universität Wien - Prof. Dr. Norbert Richard Wolf, Universität Würzburg

\subsection{Stiftungsrat}

Vorsitzender: Ltd. Min.Rat Dr. Herberger, Ministerium für Wissenschaft, Forschung und Kunst Baden-Württemberg

Stellvertreter: Reg.Dir. Dr. Pusch, Bundesministerium für Bildung, Wissenschaft, Forschung und Technologie

Dir. Peter Roschy, Vorsitzender des Vereins der Freunde des Instituts für deutsche Sprache, Mannheim - LR I Lehne, Auswärtiges Amt - Bürgermeister Lothar Mark, Stadt Mannheim - Prof. Dr. Heinrich Löffler, Universität Basel - Min.Rat Schrade, Ministerium für Wissenschaft, Forschung und Kunst Baden-Württemberg

\subsection{Direktor}

Prof. Dr. Gerhard Stickel

\subsection{Leitungskollegium}

Direktor: Prof. Dr. Gerhard Stickel - Abteilungsleiter: Prof. Dr. Werner Kallmeyer (Pragmatik) - Prof. Dr. Hartmut Schmidt (Lexik) - Prof. Dr. Bruno Strecker (komm. Leiter Grammatik) (bis zum 30.9.1998) - Prof. Dr. Gisela Zifonun (ab 1.10.1998) - Mitarbeiterausschussvorsitzende: PD Dr. Ulrike Haß-Zumkehr

\subsection{Mitarbeiterausschuss}

Vorsitzende: PD Dr. Ulrike Haß-Zumkehr

Stellverter: Dr. Wilfried Schütte

Dr. Renate Pasch, Dipl.-Inf. Eric Seubert, Dr. Doris Steffens, Prof. Dr. Bruno Strecker, Dr. Annette Trabold

\subsection{Internationaler Wissenschaftlicher Rat}

Prof. Dr. Werner Abraham, Paterswolde, Niederlande - Prof. Dr. Gerhard Augst, Siegen - Prof. Dr. Peter Bassola, Szeged, Ungarn - Prof. Dr. Hen- 
ning Bergenholtz, Arhus, Dänemark - Prof. Dr. Anne Betten, Salzburg, Österreich - Prof. Dr. Daniel Bresson, Aix-en-Provence, Frankreich Prof. Dr. Harald Burger, Zürich, Schweiz - Prof. Dr. Dieter Cherubim, Göttingen - Prof. Dr. Michael Clyne, Clayton, Australien - Prof. Dr. Martine Dalmas, Metz, Frankreich - Prof. Dr. Martin Durrell, Manchester, Großbritannien -Prof. Dr. Veronika Ehrich, Tübingen - Prof. Dr. Ulla Fix, Leipzig - Prof. Dr. Csaba Földes, Veszprem, Ungarn - Prof. Dr. Gertrud Greciano, Strasbourg, Frankreich - Prof. Dr. Franciszek Grucza, Warschau, Polen - Prof. Dr. Walter Haas, Freiburg, Schweiz - Prof. Dr. John Hawkins, Los Angeles, USA - Prof. Dr. Hans Jürgen Heringer, Augsburg - Prof. Dr. Regina Hessky, Budapest, Ungarn - Prof. Dr. Rudolf Hoberg, Darmstadt - Prof. Dr. Werner Holly, Chemnitz - Prof. Dr. Ludwig Jäger, Aachen - Prof. Dr. Alan Kirkness, Auckland, Neuseeland - Prof. Dr. Gottfried Kolde, Genève, Schweiz - Prof. Dr. Jarmo Korhonen, Helsinki, Finnland - Prof. Dr. Oddleif Leirbukt, Bergen, Norwegen - Prof. Dr. Karl Lepa, Tartu, Estland - Prof. Dr. Angelika Linke, Zürich, Schweiz - Prof. Dr. Henrik Nikula, Vaasa, Finnland - Professor Dr. Inger Rosengren, Lund, Schweden - Prof. Dr. Carlo Serra Borneto, Roma, Italien - Prof. Dr. Eugeniusz Tomiczek, Wroclaw, Polen - Prof. Dr. Michael Townson, Dublin, Irland Prof. Dr. Vural Ülkü, Mersin, Türkei - Prof. Dr. Sigurd Wichter, Göttingen - Prof. Dr. M. I. Teresa Zurdo, Madrid, Spanien

\subsection{Beiräte}

Beirat „Deutsches Fremdwörterbuch“

Prof. Dr. Oskar Reichmann, Heidelberg - Dr. Heino Speer, Heidelberg

Beirat „Erklärende Synonymik“

Prof. Dr. Herbert Ernst Wiegand, Heidelberg - Prof. Dr. Peter Rolf Lutzeier, London - Prof. Dr. Georg Meggle, Leipzig

Beirat ,Rhetorik“"

Prof. Dr. Barbara Sandig, Saarbrücken (Vorsitzende) - Prof. Dr. Walter Dieckmann, Berlin - Prof. Dr. Carl Friedrich Graumann, Heidelberg Prof. Dr. Elisabeth Gülich, Bielefeld - Prof. Dr. Werner Holly, ChemnitzZwickau

\section{Besondere Nachrichten}

\subsection{Personalia}

- Seit Anfang März 1998 ist Herr Prof. Dr. Werner Kallmeyer (Abteilung Pragmatik) stellvertretender Direktor des Instituts. Nach $\S 8$ Abs. 8 der Satzung vertritt der stellvertretende Direktor den Direktor „bei dessen Verhinderung in allen Leitungsgeschäften". 
- Seit 1. Oktober 1998 ist Frau Prof. Dr. Gisela Zifonun (vorher FU Berlin) Leiterin der Abteilung Grammatik. Sie wurde für diese Aufgabe in einem gemeinsamen Berufungsverfahren von IDS und Universität Mannheim gewonnen.

- In ihrer Zeit als Gastwissenschaftlerin am Institut für wissensbasierte Systeme der IBM in Heidelberg erfand die IDS-Mitarbeiterin Frau Dr. Angelika Storrer gemeinsam mit Frau Dr. Ulrike Schwall ein „Computersystem und Verfahren zur automatischen Analyse eines Textes“. Die IBM hat die Erfindung zum Patent angemeldet, das seit dem 30. April 1998 vom Deutschen Patentamt unter der Kennung DE 4311211 C2 geführt wird. IBM belohnte die Leistung der beiden Sprachwissenschaftlerinnen mit dem „Patent Application Invention Achievement Award“.

- Prof. Dr. Dieter Herberg (Abteilung Lexik) wurde am 16. Juni 1998 zum Mitglied der Kommission für die deutsche Rechtschreibung berufen.

- Am 11. Mai 1998 starb der langjährige Mitarbeiter des IDS Dr. Wolfgang Klein im Alter von 57 Jahren.

\subsection{Vermischtes}

- Treffen mit Vetretern der Industrie- und Handelskammer

Der Betriebswirtschaftliche Ausschuss der Industrie- und Handelskammer Rhein-Neckar war im September Gast des IDS. Das Treffen stand unter dem Rahmenthema „Sprache - Erfolgsfaktor in der Wirtschaft“. Nach der Begrüßung und Einführung in die Arbeit des IDS durch Prof. Dr. Werner Kallmeyer, folgte eine Präsentation von Mitarbeitern der Abteilung Pragmatik, die die Bedeutung von Sprache/Kommunikation in der Wirtschaft und für die Wirtschaft an unterschiedlichen Aspekten verdeutlichten.

Die kleine Präsentationsreihe wurde eröffnet von Dr. Reinhold Schmitt. Dieser zeigte den speziellen Beitrag, den die Sprachwissenschaft bei der Erstellung verständlicher Gebrauchsanweisungen leisten kann. Prof. Dr. Reinhard Fiehler stellte ein Konzept von Kommunikationstraining vor, das auf der Grundlage authentischer Gespräche basiert, die im konkreten Arbeitskontext derjenigen aufgezeichnet werden, die geschult werden sollen. Dr. Thomas Spranz-Fogasy schloss mit einem Vortrag zu Tücken und Schwierigkeiten, die berücksichtigt werden müssen, wenn sich ein Manager in interkultureller Kommunikation fortbilden will. Aufgrund des fruchtbaren Gedankenaustauschs kam man überein, den Austausch zwischen Wirtschaft und Sprachwissenschaft auch künftig fortzusetzen.

- Britisch-deutsches Forschungsprojekt

In einem auf eine Laufzeit von drei Jahren angelegten britisch-deutschen Forschungsprojekt sollen vergleichend Einstellungen zum Prozess der europäischen Vereinigung untersucht und dargestellt werden. Das Projekt hat 
den Titel 'Haltungen zu Europa-Attitudes towards Europe'. Der deutschen Arbeitsgruppe gehören an Prof. Dr. Dieter Herberg, Dr. phil. habil. Fritz Hermanns, Dr. Heidrun Kämper und Dr. Wolfgang Teubert vom IDS. In England ist das Projekt am German Department der University of Durham etabliert und wird dort geleitet von Prof. Dr. Collin Good und Dr. phil. habil. Andreas Musolff. Das Projekt wird gefördert vom Deutschen Akademischen Austauschdienst und dem British Council. Ein erstes Arbeitsgespräch fand am 26. Und 27. Oktober 1998 in der University of Durham statt.

Grundlage des Projekts sind Pressetexte, offizielle Dokumente, Interviews, Reden, Parteiprogramme, Protokolle von Parlamentsdebatten u. ä., um die wichtigsten Positionen der Europa-Diskussion in beiden Ländern herauszuarbeiten, wie sie sich in Metaphern, in Slogans und in Schlüsselwörtern ausdrücken.

- Zwischenstaatliche Kommission für deutsche Rechtschreibung

Die Zwischenstaatliche Kommission für deutsche Rechtschreibung legte Ende 1997 ihren ersten Bericht vor in der Absicht, die Diskussion zu versachlichen und die Akzeptanz gegenüber der Neuregelung zu erhöhen. Der Bericht wurde etwa 30 gesellschaftlich relevanten Verbänden und Institutionen in Deutschland, Liechtenstein, Österreich und der Schweiz mit der Bitte um Stellungnahme zugeschickt. Die Kommission lud darüber hinaus die Verbände am 23. Januar 1998 nach Mannheim ein, wertete die mündlichen und schriftlichen Stellungnahmen aus und übergab den überarbeiteten Bericht den Kultusbehörden. Die Entscheidung der politisch Verantwortlichen, jetzt keine Änderungen an der Neuregelung vorzunehmen, veranlasste Prof. Dr. Peter Eisenberg, aus der Kommission auszutreten. Die Sprachdidakterin Prof. Dr. Mechthild Dehn (Hamburg) und der Orthographieforscher Prof. Dr. Dieter Herberg (Mannheim) wurden neu in die Kommission berufen. Die Arbeitssitzungen dienten der systematischen Klärung von Zweifelsfragen, in mehreren Beratungen mit Vertretern von Wörterbuchverlagen wurden Interpretations- und Darstellungsprobleme gelöst. Mit dem am 14. Juli 1998 verkündeten einstimmigen Urteil sorgte das Bundesverfassungsgericht in Karlsruhe für Rechtssicherheit

\section{Personalstärke, Anschrift, finanzielle Angaben}

9.1 Personalstärke (Stand: 1.11.1998)

Mitarbeiter (einschl. Teilzeit- und Projektmitarbeiter):

wissenschaftliche Angestellte:

(davon beurlaubt: 1)

Verwaltungs-/technische Angestellte:

Projekt-Mitarbeiter:

Insgesamt: 


\subsection{Anschrift}

Institut für deutsche Sprache

R5, 6-13

D-68 161 Mannheim

Postanschrift:

Postfach 101621

D-68016 Mannheim

Telefon (0621) 1581-0

Telefax (0621) 1581-200

E-Mail: vorstand@ids-mannheim.de

internet: http://www.ids-mannheim.de

9.3 Haushalt des Instituts im Berichtsjahr

\section{Einnahmen:}

Ministerium für Wissenschaft, Forschung und

Kunst Baden-Württemberg

Bundesministerium für Bildung, Wissenschaft,

Forschung und Technologie

Stadt Mannheim

eigene Einnahmen

DM 6.684.000,-

DM 6.684.000,-

DM 17.000,-

DM 127.000,-

DM 13.512.000,-

Ausgaben:

Personalausgaben

Sachausgaben

Zuweisungen, Zuschüsse

Investitionen

\section{Projektmittel:}

\section{Bund:}

SERGES

Sachmittel

Land Baden-Württemberg:

$\frac{\mathrm{DM}}{\mathrm{DM}}-\frac{6.305,-}{6.305,-}$

\section{ALIGNMENT}

Personalmittel

VW-Stiftung:

Deutsch-chinesisches Verbvalenz-Wörterbuch

Personalmittel

Sachmittel
DM 10.896.000,-

DM 2.538.000,-

DM $\quad 14.000,-$

DM 64.000,-

DM 13.512.000,-

\begin{tabular}{ll} 
DM & $27.300,-$ \\
\hline $\mathrm{DM}$ & $27.300,-$
\end{tabular}

\begin{tabular}{lr} 
DM & $14.550,-$ \\
DM & $8.100,-$ \\
\hline DM & $22.650,-$
\end{tabular}


Datenbank gesprochenes Deutsch

Personalmittel

\begin{tabular}{lr} 
DM & $12.090,-$ \\
DM & $138.078,-$ \\
\hline DM & $150.168,-$
\end{tabular}

Sachmittel

Kommission der EU:

\section{PAROLE II}

Personalmittel

DM 27.540,-

Sachmittel

$\underline{\mathrm{DM}} \quad 98.630,-$

ELAN

Personalmittel

DM 126.170,-

Sachmittel

\begin{tabular}{lr} 
DM & $18.150,-$ \\
DM & $102.597,-$ \\
\hline DM & $120.747,-$
\end{tabular}

SIMPLE

Personalmittel

\begin{tabular}{ll} 
DM & $51.277,-$ \\
DM & $18.853,-$ \\
\hline DM & $70.130,-$
\end{tabular}

Sonstige:

Kommunikation in Meetings/SAP

Sachmittel

Neuauflage „Handbuch für Sprach-Kultur/

Robert-Bosch-Stiftung“

Sachmittel

$\begin{array}{ll}\mathrm{DM} & 48.150,- \\ \mathrm{DM} & 48.150,-\end{array}$

$\begin{array}{ll}\text { DM } & 22.500,- \\ \text { DM } & 22.500,-\end{array}$

ARC-Projekt/DAAD

Sachmittel

$\begin{array}{ll}\mathrm{DM} & 5.300,- \\ \mathrm{DM} & 5.300,-\end{array}$

Summe der Projektmittel

DM 599.420,-

Institutioneller Haushalt

DM 13.512.000,-

DM 14.111.420,-

\section{Veröffentlichungen im Jahre 1998}

JAHRBÜCHER DES INSTITUTS FÜR DEUTSCHE SPRACHE

Verlag Walter de Gruyter, Berlin/New York

Redaktion: Franz Josef Berens

Kämper, Heidrun/Schmidt, Hartmut (Hg.): Das 20. Jahrhundert. Sprachgeschichte - Zeitgeschichte. Jahrbuch 1997 des Instituts für deutsche Sprache. 446 S. 
STUDIEN ZUR DEUTSCHEN SPRACHE

Forschungen des Instituts für deutsche Sprache

Hg. von Hartmut Günther, Reinhard Fiehler und Bruno Strecker

ab Band 13: Bruno Strecker, Reinhard Fiehler und Hartmut Schmidt

Gunter Narr Verlag, Tübingen

Band 10: Bresson, Daniel/Kubczak, Jacqueline (Hg.): Abstrakte Nomina. Vorarbeiten zu ihrer Erfassung in einem zweisprachigen syntagmatischen Wörterbuch. $300 \mathrm{~S}$.

Band 11: Teubert, Wolfgang (Hg.): Neologie und Korpus. $170 \mathrm{~S}$.

Band 12: Storrer, Angelika/Harriehausen, Bettina (Hg.): Hypermedia für Lexikon und Grammatik. 275 S.

Band 13: Haß-Zumkehr, Ulrike: „Wie glaubwürdige Nachrichten versichert haben". Formulierungstraditionen in Zeitungsnachrichten des 17. bis 20. Jahrhunderts. $240 \mathrm{~S}$.

Band 14: Berend, Nina: Sprachliche Anpassung. Eine soziolinguistischdialektologische Untersuchung zum Rußlanddeutschen. $253 \mathrm{~S}$.

\section{STUDIENBIBLIOGRAPHIEN SPRACHWISSENSCHAFT}

Im Auftrag des Instituts für deutsche Sprache hg. von Manfred W. Hellmann.

Julius Groos Verlag Heidelberg

Band 23: Herberg, Dieter/Kinne, Michael: Neologismen. 48 S. Diskette.

Band 24: Honnef-Becker, Irmgard/Kühn, Peter: Deutsch als Fremdsprache (Sonderband) $150 \mathrm{~S}$. Diskette.

Band 25: Hoffmann, Ludger: Grammatik der gesprochenen Sprache. 56 S. Diskette.

Band 26: Fluck, Hans R.: Fachsprachen und Fachkommunikation. $50 \mathrm{~S}$. Diskette.

PHONAI - Texte und Untersuchungen zum gesprochenen Deutsch

Hg. vom Deutschen Spracharchiv im Institut für deutsche Sprache Max Niemeyer Verlag, Tübingen

Band 43: Barden, Birgit/Großkopf, Beate: Sprachliche Akkomodation und soziale Integration. Sächsische Übersiedler und Übersiedlerinnen im rhein-/moselfränkischen und alemannischen Sprachraum. XV/404 S.

\section{DEUTSCHE SPRACHE}

Im Auftrag des Instituts für deutsche Sprache

$\mathrm{Hg}$. von Siegfried Grosse (Geschäftsführung), Hans-Werner Eroms, Gisela Harras und Gerhard Stickel

Redaktion: Franz Josef Berens

Erich Schmidt Verlag, Berlin

Jahrgang 1998: 4 Hefte 


\section{SPRACHREPORT}

Informationen und Meinungen zur deutschen Sprache

Hg. vom Institut für deutsche Sprache

Jahrgang 1998: 4 Hefte

VERÖFFENTLICHUNGEN IM EIGENVERLAG

amades - Arbeitspapiere und Materialien zur deutschen Sprache

Hg. vom Institut für deutsche Sprache

Band 0: Hoppe, Gabriele: Herausbildung und Integration des Submusters ETHNIKA + -(o)phone/-(o)phonie im Französischen und Stellung des analogen Musters im Lehnwortbildungssystem des Deutschen. 92 S. Diskette.

Teubert, Wolfgang/Tognini-Bonelli, Elena/Volz, Norbert (Hg.): Proceedings of the Third European Seminar „Translation Equivalence“, Montecatini Terme, Italy, October 16-18, 1997. Birmingham/Mannheim: The Tuscan Word Centre/TELRI Association e. V./Institut für deutsche Sprache. $276 \mathrm{~S}$.

Teubert, Wolfgang/Plöger, Kirsten (Hg.): TELRI Final Report. Compiled and edited by Wolfgang Teubert and Kirsten Plöger. Birmingham/Mannheim: The Tuscan Word Centre/TELRI Association e. V./Institut für deutsche Sprache. 124 S. + CD-ROM.

Erjavec, Tomaz/Lawson, Ann/Romary, Laurent (Hg.): East meets West A Compendium of Multilingual Resources. Mannheim: TELRI Association e. V./Institut für deutsche Sprache. 2 CD-ROMs + Beiheft [8 S.]. 